\title{
DESINGULARIZATION OF QUIVER GRASSMANNIANS FOR GENTLE ALGEBRAS
}

\author{
XINHONG CHEN AND MING LU ${ }^{\dagger}$
}

\begin{abstract}
Following [20, a desingularization of arbitrary quiver Grassmannians for finite dimensional Gorenstein projective modules of 1-Gorenstein gentle algebras is constructed in terms of quiver Grassmannians for their Cohen-Macaulay Auslander algebras.
\end{abstract}

\section{INTRODUCTION}

1.1. Gorenstein projective mdules. The concept of Gorenstein projective modules over any ring can be dated back to 4, where Auslander and Bridger introduced the modules of $G$-dimension zero over two-sided noetherian rings, and formed by Enochs and Jenda [26]. This class of modules satisfy some good stable properties, becomes a main ingredient in the relative homological algebra, and widely used in the representation theory of algebras and algebraic geometry, see e.g. [4, 6, 26, 12, 32, 8. It also plays as an important tool to study the representation theory of Gorenstein algebra, see e.g. [6, 12, 32].

1.2. Gorenstein algebras. Gorenstein algebra $\Lambda$, where by definition $\Lambda$ has finite injective dimension both as a left and a right $\Lambda$-module, is inspired from commutative ring theory. A fundamental result of Buchweitz [12] and Happel [32] states that for a Gorenstein algebra $\Lambda$, the singularity category is triangle equivalent to the stable category of the Gorenstein projective (also called (maximal) Cohen-Macaulay) $\Lambda$-modules, which generalized Rickard's result [45] on self-injective algebras.

1.3. Cohen-Macaulay Auslander algebras. For any Artin algebra $\Lambda$, denote by Gproj $\Lambda$ its subcategory of Gorenstein projective modules. If Gproj $\Lambda$ has only finitely many isoclasses of indecomposable objects, then $\Lambda$ is called CM-finite. In this case, inspired by the definition of Auslander algebra, the Cohen-Macaulay Auslander algebra (also called the relative Auslander algebra) is defined to be $\operatorname{End}_{\Lambda}\left(\bigoplus_{i=1}^{n} E_{i}\right)^{o p}$, where $E_{1}, \ldots, E_{n}$ are all pairwise nonisomorphic indecomposable Gorenstein projective modules, [7, 8, 39]. A CM-finite algebra $\Lambda$ is Gorenstein if and only if gl. $\operatorname{dim} \operatorname{Aus}(\operatorname{Gproj} \Lambda)<\infty$, 39, 8. Pan proves that for any two Gorenstein Artin algebras $A$ and $B$ which are CM-finite, if $A$ and $B$ are derived equivalent, then their Cohen-Macaulay Auslander algebras are also derived equivalent [43.

1.4. Gentle algebras. As an important class of Gorenstein algebras [31, gentle algebras were introduced in [3] as appropriate context for the investigation of algebras derived equivalent to hereditary algebras of type $\tilde{\mathbb{A}}_{n}$. The gentle algebras which are trees are precisely the algebras derived equivalent to hereditary algebras of type $\mathbb{A}_{n}$, see [2]. It is interesting to notice that the class of gentle algebras is closed under derived equivalence, [48. For singularity categories of gentle algebras, Kalck determines their singularity category by finite products

2000 Mathematics Subject Classification. 13F60, 14M15, 16G20.

Key words and phrases. Desingularization, Quiver Grassmannians, Cohen-Macaulay Auslander algebra, Gentle algebra.

${ }^{\dagger}$ Corresponding author. 
of $d$-cluster categories of type $\mathbb{A}_{1}$ 34]. From [34, it is easy to see that Gentle algebras are CM-finite, which inspires us to study the properties of the Cohen-Macaulay Auslander algebras of Gentle algebras [23. Moreover, many important algebras are gentle, such as tilted algebras of type $\mathbb{A}_{n}$, algebras derived equivalent to $\mathbb{A}_{n}$-configurations of projective lines [13] and also the cluster-tilted algebras of type $\mathbb{A}_{n}[11], \tilde{\mathbb{A}}_{n}[1]$.

1.5. Quiver Grassmannians. Quiver Grassmannians were first introduced by CrawleyBoevey [24, Schofield [47] to study the generic properties of quiver representations. They are projective varieties parametrizing subrepresentations of a quiver representation and Reineke shows in [4] that every projective variety can be realized as a quiver Grassmannian. It was observed in [15] that these varieties play an important role in the additive categorification of (quantum) cluster algebra theory [30] since cluster variables can be described in terms of the Euler characteristic of quiver Grassmannians, cf. for example [15, 16, 25, 41. Subsequently, specific classes of quiver Grassmannians were studied by several authors, in particular, the varieties of subrepresentations of exceptional quiver representations since they are smooth projective varieties, see e.g. [17.

In [18, 19, Cerulli-Feigin-Reineke initiated a systematic study of (singular) quiver Grassmannians of Dynkin quivers, starting from the surprising observation that the type $\mathbb{A}$ degenerate flag varieties studies in [27, 28, 29] are of this form. An important aspect of their work is the construction of desingularizations for the Dynkin type, namely a desingularization of arbitrary quiver Grassmannians for representations of Dynkin quivers is constructed in terms of quiver Grassmannians for an algebra derived equivalent to the Auslander algebra of the quiver, which is done in 20] generalizing [29] for the type $\mathbb{A}$ degenerate flag varieties. After that, they link these desingularizations to a construction by Hernandez-Leclerc [33, which has been generalizeed by Leclerc-Plamondon [38] and further generalized by KellerScherotzke [36, 37]. The desingularization constructed in [37] is the desingularization map for graded quiver varieties introduced by Nakajima [40, 41], and this generalized [20] to much more general situations and in particular for all modules over the repetitive algebra of an arbitrary iterated tilted algebra of Dynkin type.

1.6. Main result. In this paper, we consider the quiver Grassmannians of arbitrary Gorenstein projective modules over 1-Gorenstein gentle algebras $\Lambda$. Inspired by [20], we use quiver Grassmannians over the Cohen-Macaulay Auslander algebra $\Gamma$ of $\Lambda$ to construct a desingularization of the quiver Grassmannians of arbitrary Gorenstein projective modules over $\Lambda$.

The paper is organized as follows. In section 2, we collect some standard material on gentle algebras, Gorenstein algebras and quiver Grassmannians. In Section 3, we define the key functor $\Phi: \bmod \Lambda \rightarrow \bmod \Gamma$, and prove that for any Gorenstein projective $\Lambda$-module $M$, the quiver Grassmannians of $\Phi(M)$ is smooth with irreducible and equidimensional connected components. In Section 4, we use the technique of [20] and the results of the previous sections to construct the desingularizations of quiver Grassmannians. Finally, in Section 5 we close with some examples of desingularizations.

Acknowledgments. This work is inspired by some discussions with the first author's supervisor Bin Zhang. The authors thank him very much.

The first author(X. Chen) was supported by the Fundamental Research Funds for the Central Universities A0920502051411-45.

The corresponding author(M. Lu) was supported by the National Natural Science Foundation of China (Grant No. 11401401). 


\section{Preliminaries}

Let $K$ be an algebraically closed field. For a $K$-algebra, we always mean a basic finite dimensional associative $K$-algebra. For an additive category $\mathcal{A}$, we denote by ind $\mathcal{A}$ the isomorphism classes of indecomposable objects in $\mathcal{A}$.

Let $Q$ be a quiver and $\langle I\rangle$ an admissible ideal in the path algebra $K Q$ which is generated by a set of relations $I$. Denote by $(Q, I)$ the associated bound quiver. For any arrow $\alpha$ in $Q$ we denote by $s(\alpha)$ its starting vertex and by $t(\alpha)$ its ending vertex. An oriented path (or path for short) $p$ in $Q$ is a sequence $p=\alpha_{1} \alpha_{2} \ldots \alpha_{r}$ of arrows $\alpha_{i}$ such that $t\left(\alpha_{i}\right)=s\left(\alpha_{i-1}\right)$ for all $i=2, \ldots, r$.

2.1. Gentle algebras. We first recall the definition of special biserial algebras and of gentle algebras.

Definition 2.1 ([49]). The pair $(Q, I)$ is called special biserial if it satisfies the following conditions.

- Each vertex of $Q$ is starting point of at most two arrows, and end point of at most two arrows.

- For each arrow $\alpha$ in $Q$ there is at most one arrow $\beta$ such that $\alpha \beta \notin I$, and at most one arrow $\gamma$ such that $\gamma \alpha \notin I$.

Definition $2.2([3])$. The pair $(Q, I)$ is called gentle if it is special biserial and moreover the following holds.

- The set I is generated by zero-relations of length 2 .

- For each arrow $\alpha$ in $Q$ there is at most one arrow $\beta$ with $t(\beta)=s(\alpha)$ such that $\alpha \beta \in I$, and at most one arrow $\gamma$ with $s(\gamma)=t(\alpha)$ such that $\gamma \alpha \in I$.

A finite dimensional algebra $A$ is called special biserial (resp., gentle), if it has a presentation as $A=K Q /\langle I\rangle$ where $(Q, I)$ is special biserial (resp., gentle).

2.2. Singularity categories and Gorenstein algebras. Let $\Gamma$ be a finite dimensional $K$ algebra. Let $\bmod \Gamma$ be the category of finitely generated left $\Gamma$-modules. For an arbitrary $\Gamma$-module $\Gamma_{\Gamma} X$, we denote by proj. $\operatorname{dim}_{\Gamma} X$ (resp. inj. $\operatorname{dim}_{\Gamma} X$ ) the projective dimension (resp. the injective dimension) of the module ${ }_{\Gamma} X$. A $\Gamma$-module $G$ is Gorenstein projective, if there is an exact sequence

$$
P^{\bullet}: \cdots \rightarrow P^{-1} \rightarrow P^{0} \stackrel{d^{0}}{\rightarrow} P^{1} \rightarrow \cdots
$$

of projective $\Gamma$-modules, which stays exact under $\operatorname{Hom}_{\Gamma}(-, \Gamma)$, and such that $G \cong \operatorname{Ker} d^{0}$. We denote by $\operatorname{Gproj}(\Gamma)$ the subcategory of Gorenstein projective $\Gamma$-modules.

Definition $2.3([5,6])$. A finite dimensional algebra $\Gamma$ is called a Gorenstein algebra if $\Gamma$ satisfies inj. $\operatorname{dim} \Gamma_{\Gamma}<\infty$ and inj. $\operatorname{dim}_{\Gamma} \Gamma<\infty$.

Observe that for a Gorenstein algebra $\Gamma$, we have inj. $\operatorname{dim}_{\Gamma} \Gamma=\operatorname{inj} \cdot \operatorname{dim} \Gamma_{\Gamma}$, [32, Lemma 6.9]; the common value is denoted by G. $\operatorname{dim} \Gamma$. If G. $\operatorname{dim} \Gamma \leq d$, we say that $\Gamma$ is $d$-Gorenstein.

Theorem 2.4 ([12, 26]). Let $\Gamma$ be an artin algebra and let $d \geq 0$. Then the following statements are equivalent:

(1) the algebra $\Gamma$ is $d$-Gorenstein;

(2) $\operatorname{Gproj}(\Gamma)=\Omega^{d}(\bmod \Gamma)$, where $\Omega$ is the syzygy functor.

In this case, $A$ module $G$ is Gorenstein projective if and only if there is an exact sequence $0 \rightarrow G \rightarrow P^{0} \rightarrow P^{1} \rightarrow \cdots$ with each $P^{i}$ projective. 
For an algebra $\Gamma$, the singularity category of $\Gamma$ is defined to be the quotient category $D_{s g}^{b}(\Gamma):=D^{b}(\Gamma) / K^{b}($ proj. $\Gamma)[12,32,42]$. Note that $D_{s g}^{b}(\Gamma)$ is zero if and only if gl. $\operatorname{dim} \Gamma<\infty$ 32 .

Theorem 2.5. [12, 32] Let $\Gamma$ be a Gorenstein algebra. Then $\operatorname{Gproj}(\Gamma)$ is a Frobenius category with the projective modules as the projective-injective objects. The stable category $\mathrm{Gproj}(\Gamma)$ is triangle equivalent to the singularity category $D_{s g}^{b}(\Gamma)$ of $\Gamma$.

An algebra is of finite Cohen-Macaulay type, or simply, CM-finite, if there are only finitely many isomorphism classes of indecomposable finitely generated Gorenstein projecitve modules. Clearly, $A$ is CM-finite if and only if there is a finitely generated module $E$ such that $\operatorname{Gproj} A=\operatorname{add} E$. In this way, $E$ is called to be a Gorenstein projective generator. If gl. $\operatorname{dim} A<\infty$, then Gproj $A=\operatorname{proj} . A$, so $A$ is CM-finite. If $A$ is self-injective, then Gproj $A=\bmod A$, so $A$ is CM-finite if and only if $A$ is representation finite.

Let $A$ be a CM-finite algebra, $E_{1}, \ldots, E_{n}$ all the pairwise non-isomorphic indecomposable Gorenstein projective $A$-modules. Put $E=\oplus_{i=1}^{n} E_{i}$. Then $E$ is a Gorenstein projective generator. We call $\operatorname{Aus}(\operatorname{Gproj} A):=\left(\operatorname{End}_{A} E\right)^{o p}$ the Cohen-Macaulay Auslander algebra (also called relative Auslander algebra) of $A$.

Lemma 2.6 ([39]). Let $A$ be a CM-finite Artin algebra. Then we have the following:

(i) $\operatorname{gl} \operatorname{dim} \operatorname{Aus}(\operatorname{Gproj} A)=0$ if and only if $A$ is semisimple.

(ii) $\operatorname{gl} \cdot \operatorname{dim} \operatorname{Aus}(\operatorname{Gproj} A)=1$ if and only if $\mathrm{gl} \cdot \operatorname{dim} A=1$.

(iii) gl. $\operatorname{dim} \operatorname{Aus}(\operatorname{Gproj} A)=2$ if and only if either

(a) $\operatorname{Gproj} A=$ proj. $A$ and gl. $\operatorname{dim} A=2$, or

(b) Gproj $A \neq$ proj. $A$ and $A$ is a Gorenstein algebra with $\mathrm{G}$. $\operatorname{dim} A \leq 2$.

(iv) If G. $\operatorname{dim} A \geq 3$, then:

$$
\text { gl. } \operatorname{dim} \operatorname{Aus}(\operatorname{Gproj} A)=\text { G. } \operatorname{dim} A .
$$

(v) $A$ is Gorenstein if and only if Cohen-Macaulay Auslander algebra Aus(Gproj A) has finite global dimension.

Geiß and Reiten [31] have shown that gentle algebras are Gorenstein algebras. So their Cohen-Macaulay Auslander algebras have finite global dimensions.

The singularity category of a gentle algebra is characterized by Kalck in [34, we recall it as follows. For a gentle algebra $\Lambda=K Q /\langle I\rangle$, we denote by $\mathcal{C}(\Lambda)$ the set of equivalence classes (with respect to cyclic permutation) of repetition-free cyclic paths $\alpha_{1} \ldots \alpha_{n}$ in $Q$ such that $\alpha_{i} \alpha_{i+1} \in I$ for all $i$, where we set $n+1=1$. Moreover, we set $l(c)$ for the length of a cycle $c \in \mathcal{C}(\Lambda)$, i.e. $l\left(\alpha_{1} \ldots \alpha_{n}\right)=n$.

For every arrow $\alpha \in Q_{1}$, there is at most one cycle $c \in \mathcal{C}(\Lambda)$ containing it. We define $R(\alpha)$ to be the left ideal $\Lambda \alpha$ generated by $\alpha$. It follows from the definition of gentle algebras that this is a direct summand of the radical $\operatorname{rad} P_{s(\alpha)}$ of the indecomposable projective $\Lambda$-module $P_{s(\alpha)}=\Lambda e_{s(\alpha)}$, where $e_{s(\alpha)}$ is the idempotent corresponding to $s(\alpha)$. In fact, all radical summands of indecomposable projectives arise in this way.

Theorem 2.7 (34]). Let $\Lambda=K Q /\langle I\rangle$ be a gentle algebra. Then

(i) ind $\operatorname{Gproj}(\Lambda)=$ ind proj. $\Lambda \bigcup\left\{R\left(\alpha_{1}\right), \ldots, R\left(\alpha_{n}\right) \mid c=\alpha_{1} \cdots \alpha_{n} \in \mathcal{C}(\Lambda)\right\}$.

(ii) There is an equivalence of triangulated categories

$$
D_{s g}^{b}(\Lambda) \simeq \prod_{c \in \mathcal{C}(\Lambda)} \frac{D^{b}(K)}{[l(c)]}
$$

where $D^{b}(K) /[l(c)]$ denotes the triangulated orbit category, see Keller [35]. 
From Theorem 2.7, we get that

$$
\text { ind } \operatorname{Gproj}(\Lambda)=\text { ind proj. } \Lambda \bigcup\left\{R\left(\alpha_{1}\right), \ldots, R\left(\alpha_{n}\right) \mid c=\alpha_{1} \cdots \alpha_{n} \in \mathcal{C}(\Lambda)\right\} \text {. }
$$

Furthermore, from its proof, let $c \in \mathcal{C}(\Lambda)$ be a cycle, which we label as follows: $1 \stackrel{\alpha_{1}}{\longrightarrow} 2 \stackrel{\alpha_{2}}{\longrightarrow}$ $\cdots \stackrel{\alpha_{n-1}}{\longrightarrow} n \stackrel{\alpha_{n}}{\longrightarrow} 1$. Then there are short exact sequences

$$
0 \rightarrow R\left(\alpha_{i}\right) \stackrel{a_{i}}{\rightarrow} P_{i} \stackrel{b_{i}}{\rightarrow} R\left(\alpha_{i-1}\right) \rightarrow 0,
$$

for all $i=1, \ldots, n$.

A classification of indecomposable modules over gentle algebras can be deduced from work of Ringel [46] (see e.g. [14, 50]). For each arrow $\beta$, we denote by $\beta^{-1}$ the formal inverse of $\beta$ with $s\left(\beta^{-1}\right)=t(\beta)$ and $t\left(\beta^{-1}\right)=s(\beta)$. A word $w=c_{1} c_{2} \cdots c_{n}$ of arrows and their formal inverse is called a string of length $n \geq 1$ if $c_{i+1} \neq c_{i}^{-1}, s\left(c_{i}\right)=t\left(c_{i+1}\right)$ for all $1 \leq i \leq$ $n-1$, and no subword nor its inverse is in $I$. We define $\left(c_{1} c_{2} \cdots c_{n}\right)^{-1}=c_{n}^{-1} \cdots c_{2}^{-1} c_{1}^{-1}$, and $s\left(c_{1} c_{2} \cdots c_{n}\right)=s\left(c_{n}\right), t\left(c_{1} c_{2} \cdots c_{n}\right)=t\left(c_{1}\right)$. We denote the length of $w$ by $l(w)$. In addition, we also want to have strings of length 0 ; be definition, for any vertex $u \in Q_{0}$, there will be two strings of length 0 , denoted by $1_{(u, 1)}$ and $1_{(u,-1)}$, with both $s\left(1_{(u, i)}\right)=u=t\left(1_{(u, i)}\right)$ for $i=-1,1$, and we define $\left(1_{(u, i)}\right)^{-1}=1_{(u,-i)}$. We also denote by $\mathcal{S}(\Lambda)$ the set of all strings over $\Lambda=K Q /\langle I\rangle$.

A band $b=\alpha_{1} \alpha_{2} \cdots \alpha_{n-1} \alpha_{n}$ is defined to be a string $b$ with $t\left(\alpha_{1}\right)=s\left(\alpha_{n}\right)$ such that each power $b^{m}$ is a string, but $b$ itself is not a proper power of any strings. We denote by $\mathcal{B}(\Lambda)$ the set of all bands over $\Lambda$.

On $\mathcal{S}(\Lambda)$, we consider the equivalence relation $\rho$ which identifies every string $C$ with its inverse $C^{-1}$. On $\mathcal{B}(\Lambda)$, we consider the equivalence relation $\rho^{\prime}$ which identifies every string $C=c_{1} \ldots c_{n}$ with the cyclically permuted strings $C_{(i)}=c_{i} c_{i+1} \cdots c_{n} c_{1} \cdots c_{i-1}$ and their inverses $C_{(i)}^{-1}, 1 \leq i \leq n$. We choose a complete set $\underline{\mathcal{S}}(\Lambda)$ of representatives of $\mathcal{S}(\Lambda)$ relative to $\rho$, and a complete set $\underline{\mathcal{B}}(\Lambda)$ of representatives of $\mathcal{B}(\Lambda)$ relative to $\rho^{\prime}$.

Butler and Ringel showed that each string $w$ defines a unique string module $M(w)$, each band $b$ yields a family of band modules $M(b, m, \phi)$ with $m \geq 1$ and $\phi \in \operatorname{Aut}\left(K^{m}\right)$. Equivalently, one can consider certain quiver morphism $\sigma: S \rightarrow Q$ (for strings) and $\beta: B \rightarrow Q$ (for bands), where $S$ and $B$ are of Dynkin types $\mathbb{A}_{n}$ and $\tilde{A}_{n}$, respectively. Then string and band modules are given as pushforwards $\sigma_{*}(M)$ and $\beta_{*}(R)$ of indecomposable $K S$-modules $M$ and indecomposable regular $K B$-modules $R$, respectively (see e.g. [50]). Let $\underline{\operatorname{Aut}}\left(K^{m}\right)$ be a complete set of representatives of indecomposable automorphisms of $K$-spaces with respect to similarity.

Theorem 2.8 ([14). The modules $M(w)$, with $w \in \underline{\mathcal{S}}(\Lambda)$, and the modules $M(b, m, \phi)$ with $b \in \underline{\mathcal{B}}(\Lambda)$, with $b \in \underline{\mathcal{B}}(\Lambda), m \geq 1$ and $\phi \in \underline{\operatorname{Aut}}\left(K^{m}\right)$, provide a complete list of indecomposable (and pairwise non-isomorphic) $\Lambda$-modules.

In practice, a string $w$ is of form $\alpha_{1}^{\epsilon_{1}} \alpha_{2}^{\epsilon_{2}} \cdots \alpha_{n}^{\epsilon_{n}}$ for $\alpha_{i} \in Q_{1}$ and $\epsilon_{i}= \pm 1$ for all $1 \leq i \leq n$. So $w$ can be viewed as a walk in $Q$ :

$$
w: \quad 1 \stackrel{\alpha_{1}}{-} 2 \stackrel{\alpha_{2}}{\cdots} \cdots \frac{\alpha_{n-1}}{2} n \frac{\alpha_{n}}{n+1,}
$$

where $i \in Q_{0}$ are vertices of $Q$ and $\alpha_{i}$ are arrows in either directions. In this way, the equivalence relation $\rho$ induces that

$$
w: \quad 1 \stackrel{\alpha_{1}}{\longrightarrow} 2 \stackrel{\alpha_{2}}{\cdots} \cdots \frac{\alpha_{n-1}}{-\alpha_{n}} n+1,
$$


is equivalent to

$$
w^{-1}: \quad n+1 \stackrel{\alpha_{n}}{\longrightarrow} n \stackrel{\alpha_{n-1}}{2} \cdots \frac{\alpha_{2}}{2} 2 \stackrel{\alpha_{1}}{-} 1 .
$$

It is similar to interpret $\rho^{\prime}$ if $w$ is a band. We denote by $v \sim w$ for two strings $v, w$ if $v$ is equivalent to $w$ under $\rho$.

For any string $w=c_{1} \ldots c_{n}$, or $w=1_{(u, t)}$, let $u(i)=t\left(c_{i+1}\right), 0 \leq i<n$, and $u(n)=s(w)$. Given a vertex $v \in Q_{0}$, let $I_{v}=\{i \mid u(i)=v\} \subseteq\{0,1, \ldots, n\}$. Denote by $k_{v}=\left|I_{v}\right|$. We associate a vector $\left(k_{v}\right)_{v \in Q_{0}}$ to the string $w$, this vector is denoted by $\underline{\operatorname{dim}} w$, and call it the dimension vector of $w$. From [14, we get that $\underline{\operatorname{dim}} w=\underline{\operatorname{dim}} M(w)$.

2.3. Quiver Grassmannians. Quiver Grassmannians are varieties parametrizing subrepresentations of a quiver representation. They are introduced by Craw-Boevey([24]), Schofield([47]) to study the generic properties of quiver representations.

For a finite quiver $Q$, let $A=K Q / I$ be a finite dimensional algebra. We consider finite dimensional representations $M$ of $A$ over $K$, viewed either as finite dimensional left modules over the path algebra $A$, or as tuples

$$
M=\left(\left(M_{i}\right)_{i \in Q_{0}},\left(M_{\alpha}: M_{i} \rightarrow M_{j}\right)_{(\alpha: i \rightarrow j) \in Q_{1}}\right)
$$

consisting of finite dimensional $K$-vector spaces $M_{i}$ and linear maps $M_{\alpha}$.

For a representation $M$ of $A$, let $d=\underline{\operatorname{dim}} M$. We consider the affine space

$$
R_{d}(Q)=\bigoplus_{(\alpha: i \rightarrow j)} \operatorname{Hom}_{K}\left(M_{i}, M_{j}\right)
$$

its points canonically paprametrize representations of $Q$ of dimension vector of $d$. Let $R_{d}(A)$ be the subvariety of $R_{d}(Q)$ consisting of representations annihilated by $I$, where its points canonically parametrize representations of $A$ of dimension vector $d$. The reductive algebraic group $G_{d}=\prod_{i \in Q_{0}} \operatorname{GL}\left(M_{i}\right)$ acts naturally on $R_{d}(Q)$ via base change

$$
\left(g_{i}\right)_{i} \cdot\left(M_{\alpha}\right)_{\alpha}=\left(g_{j} M_{\alpha} g_{i}^{-1}\right)_{(\alpha: i \rightarrow j)},
$$

such that the orbit $\mathcal{O}_{M}$ for this action naturally corresponds to the isomorphism classes $[M]$ of representations of $Q$ of dimension vector $d$. Note that

$$
\operatorname{dim} G_{d}-\operatorname{dim} R_{d}(Q)=\sum_{i \in Q_{0}} d_{i}^{2}-\sum_{\alpha: i \rightarrow j} d_{i} d_{j}=\langle d, d\rangle_{Q} .
$$

The stabilizer under $G_{d}$ of a point $M \in R_{d}(Q)$ is isomorphic to the automorphism group $\operatorname{Aut}_{Q}(M)$ of the corresponding representation, which is a connected algebraic group of dimension $\operatorname{dim} \operatorname{End}_{Q}(M)$. In particular, we get

$$
\operatorname{dim} \mathcal{O}_{M}=\operatorname{dim} G_{d}-\operatorname{dim} \operatorname{End}_{Q}(M) .
$$

The constructions and results in the following follow [47, see also [17, 18. Additionally to the above, fix another dimension vector $e$ such that $e \leq d$ componentwise, and define the $Q_{0}$-graded Grassmannian $\operatorname{Gr}_{e}(d)=\prod_{i \in Q_{0}} \operatorname{Gr}_{e_{i}}\left(M_{i}\right)$, which is a projective homogeneous space for $G_{d}$ of dimension $\sum_{i \in Q_{0}} e_{i}\left(d_{i}-e_{i}\right)$. We define $\operatorname{Gr}_{e}^{Q}(d)$, the universal Grassmannian of $e$ dimensional subrepresentations of $d$-dimensional representations of $Q$ as the closed subvariety of $\operatorname{Gr}_{e}(d) \times R_{d}(Q)$ consisting of tuples $\left(\left(U_{i} \subseteq M_{i}\right)_{i \in Q_{0}},\left(M_{\alpha}\right)_{\alpha \in Q_{1}}\right)$ such that $M_{\alpha}\left(U_{i}\right) \subseteq U_{j}$ for all arrows $(\alpha: i \rightarrow j) \in Q_{1}$. The group $G_{d}$ acts on $\operatorname{Gr}_{e}^{Q}(d)$ diagonally, such that the projection $p_{1}: \operatorname{Gr}_{e}^{Q}(d) \rightarrow \operatorname{Gr}_{e}(d)$ and $p_{2}: \mathrm{Gr}_{e}^{Q}(d) \rightarrow R_{d}(Q)$ are $G_{d^{-}}$equivariant. In fact, the 
projection $p_{1}$ identifies $\operatorname{Gr}_{e}^{Q}(d)$ as the total space of a homogeneous bundle over $\operatorname{Gr}_{e}(d)$ of rank

$$
\sum_{(\alpha: i \rightarrow j) \in Q_{1}}\left(d_{i} d_{j}+e_{i} e_{j}-e_{i} d_{j}\right)
$$

and $\operatorname{Gr}_{e}^{Q}(d)$ is smooth and irreducible of dimension

$$
\operatorname{dim} \operatorname{Gr}_{e}^{Q}(d)=\langle e, d-e\rangle_{Q}+\operatorname{dim} R_{d}(Q) .
$$

The projection $p_{2}$ is proper, thus its image is a closed $G_{d}$-stable subvariety of $R_{d}$, consisting of representations admitting a subrepresentation of dimension vector $e$.

We define the quiver Grassmannian $\operatorname{Gr}_{e}^{Q}(M)=p_{2}^{-1}(M)$ as the fibre of $p_{2}$ over a point $M \in$ $R_{d}(Q)$; by definition, it parametrizes $e$-dimensional subrepresentations of the representation $M$.

Similarly, we define the universal quiver Grassmannian $\operatorname{Gr}_{e}^{A}(d)$ as the closed subset of $\operatorname{Gr}_{e}(d) \times R_{d}(A)$ consisting of pairs $\left(\left(U_{i} \subseteq M_{i}\right)_{i \in Q_{0}},\left(f_{\alpha}\right)_{\alpha \in Q_{1}}\right)$ such that $f_{\alpha}\left(U_{i}\right) \subseteq U_{j}$ for all arrows $\alpha: i \rightarrow j$. Using the projection $p_{2}: \operatorname{Gr}_{e}^{A}(d) \rightarrow R_{d}(A)$, we define the schemetheoretic quiver Grassmannian $\mathcal{G} r_{e}^{A}(M)=p_{2}^{-1}(M)$, thus by definition, $\operatorname{Gr}_{e}^{A}(M)$ is isomorphic to $\mathcal{G} r_{e}^{A}(M)$ endowed with the reduced structure. However, for any $A$-module $M, A$ submodules of $M$ are the same as its $K Q$-submodules, so we also denote $\operatorname{Gr}_{e}^{A}(M)$ by $\operatorname{Gr}_{e}^{Q}(M)$ or even $\operatorname{Gr}_{e}(M)$ if there is no confusion.

Theorem 2.9 ([20]). Let $Q$ be a quiver, let $M$ be a representation of $K Q$ and let e be a dimension vector for $Q$. Assume that $M$ is a representation for a quotient algebra $A=$ $K Q / I$, such that the following holds: A has global dimension at most 2, both the injective and the projective dimension of $M$ over $A$ are at most one, and $\operatorname{Ext}_{A}^{1}(M, M)=0$. Then the quiver Grassmannian $\operatorname{Gr}_{e}(M)$ is smooth (and reduced), with irreducible and equidimensional connected components.

\section{THE FUNCTOR $\Phi$}

First, we give a characterization of 1-Gorenstein gentle algebra.

Proposition 3.1. Let $\Lambda=K Q / I$ be a finite dimensional gentle algebra. Then $\Lambda$ is 1Gorenstein if and only if for any arrows $\alpha, \beta$ in $Q$ satisfying $0 \neq \beta \alpha \in I$, there exists $c \in \mathcal{C}(\Lambda)$ such that $\alpha, \beta \in c$.

Proof. First, we assume that $\Lambda$ is 1 -Gorenstein. If there are some arrows $\alpha, \beta$ such that $0 \neq \beta \alpha \in I$, however, there is no $c \in \mathcal{C}(\Lambda)$ such that $\alpha, \beta \in c$. Without losing generality, by the definition of $\mathcal{C}(\Lambda)$, we can assume that there exists one arrow $\alpha_{i}$ satisfying that there is an arrow $\alpha_{i-1}$ such that $0 \neq \alpha_{i} \alpha_{i-1} \in I$, but there is no arrow $\alpha_{i+1}$ such that $0 \neq \alpha_{i+1} \alpha_{i} \in I$. Locally, the quiver $Q$ looks as the following Figure 1 shows, where the vertices can be coincided.

Note that there exists at most one arrow $\gamma_{1}$ starts from $i$. We assume that $j_{2}, j_{3}$ are the ending vertices of the longest paths $\beta_{s} \ldots \beta_{1}$ and $\gamma_{1} \ldots \gamma_{t}$ starting from $i-1$ and $i$ respectively, with $\beta_{1} \neq \alpha_{i} ; j_{1}, j_{4}, j_{5}$ are the starting vertices of the longest paths $\eta_{1} \ldots \eta_{p}, \xi_{1} \ldots \xi_{q}, \delta_{1} \ldots \delta_{q}$ ending to $i-1, i, j_{3}$ respectively, with $\eta_{1} \neq \alpha_{i-1}, \xi_{1} \neq \alpha_{i}$ and $\delta_{1} \neq \gamma_{t}$.

Denote by $P_{i-1}$ and $P_{i}$ the indecomposable projective modules corresponding to $i-1$ and $i$ respectively, and $I_{j_{3}}$ the indecomposable injective module corresponding to $j_{3} . P_{i-1}$ is a string module with string

$$
j_{3} \stackrel{\gamma_{t}}{\longleftarrow} \cdots \stackrel{\gamma_{1}}{\longleftarrow} i \stackrel{\alpha_{i}}{\longleftarrow} i-1 \stackrel{\beta_{1}}{\longrightarrow} \cdots \stackrel{\beta_{s}}{\longrightarrow} j_{2},
$$


$P_{i}$ is a string module with string

$$
i \stackrel{\gamma_{1}}{\longrightarrow} \cdots \stackrel{\gamma_{t}}{\longrightarrow} j_{3}
$$

$I_{j_{3}}$ is a string module with string

$$
j_{1} \stackrel{\eta_{p}}{\longrightarrow} \cdots \stackrel{\eta_{1}}{\longrightarrow} i-1 \stackrel{\alpha_{i}}{\longrightarrow} i \stackrel{\gamma_{1}}{\longrightarrow} \cdots \stackrel{\gamma_{t}}{\longrightarrow} j_{3} \stackrel{\delta_{1}}{\longleftarrow} \cdots \stackrel{\delta_{u}}{\longleftarrow} j_{5} .
$$

Then there exists a short exact sequence

$$
0 \rightarrow P_{i} \rightarrow I_{j_{3}} \rightarrow M_{1} \oplus M_{2} \rightarrow 0,
$$

where $M_{1}, M_{2}$ are the string modules with their strings

$$
j_{1} \stackrel{\eta_{p}}{\longrightarrow} \cdots \stackrel{\eta_{1}}{\longrightarrow} i-1
$$

and

$$
\bullet \stackrel{\delta_{2}}{\longleftarrow} \cdots \stackrel{\delta_{u}}{\longleftarrow} j_{5}
$$

respectively. Note that $0 \neq M_{1}$ is not injective, so $\operatorname{inj}$. $\operatorname{dim} P_{i}>1$, and then $\Lambda$ is not 1 Gorenstein, a contradiction.

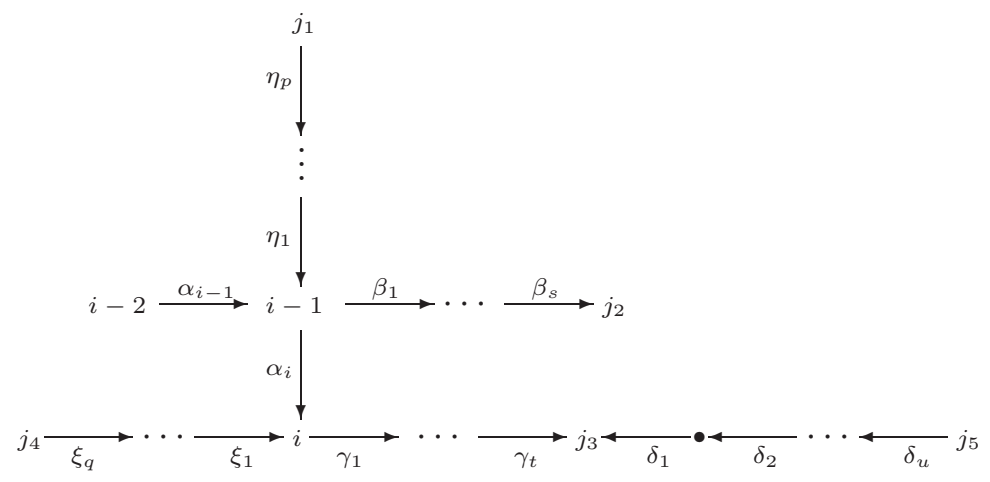

Figure 1. The quiver of $Q$.

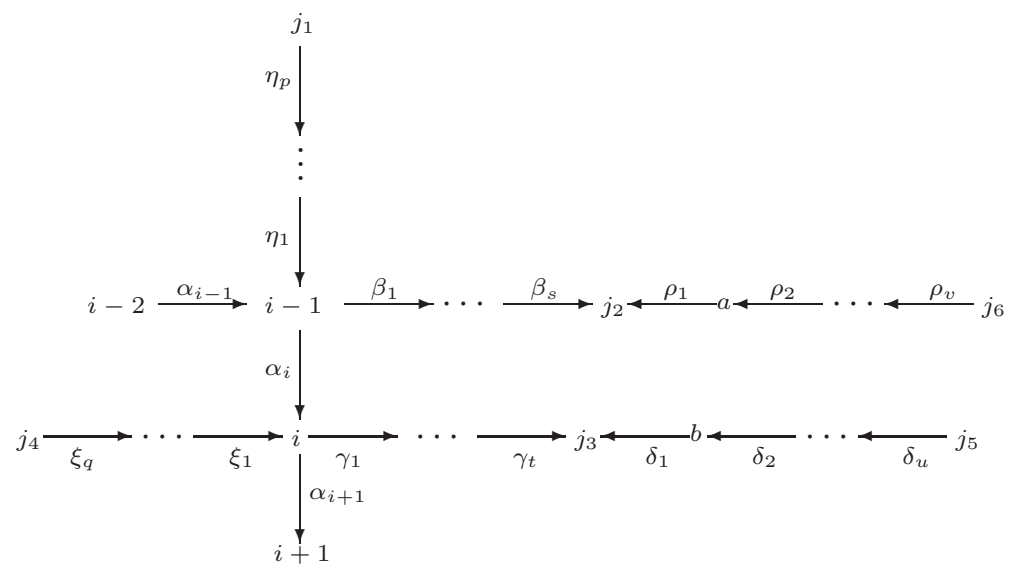

Figure 2. The quiver of $Q$. 
Conversely, for any indecomposable projective module $P$, if the corresponding vertex $i-1$ lies at a cycle $c=\alpha_{n} \ldots \alpha_{1} \in \mathcal{C}(\Lambda)$, we assume the quiver $Q$ looks as the above Figure 2 shows.

Note that the vertices $j_{1}, \ldots, j_{6}$ are defined as the above. Then there exists a short exact sequence

$$
0 \rightarrow P_{i-1} \rightarrow I_{j_{2}} \oplus I_{j_{3}} \rightarrow I_{i-1} \oplus N_{1} \oplus N_{2} \rightarrow 0,
$$

where $N_{1}, N_{2}$ are the string modules corresponding to strings

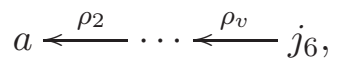

and

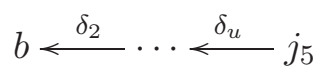

respectively.

We claim that $N_{1}$ and $N_{2}$ are injective modules. If $N_{1}$ is nonzero and not injective, then there is another arrow $\mu_{1}$ ending to $a$. Then $\rho_{1} \mu_{1} \in I$, which implies that $\rho$ belongs to a cycle $c^{\prime} \in \mathcal{C}(\Lambda)$ by the assumption. So there exists an arrow $\mu_{2}$ starting from $j_{2}$ such that $\mu_{2} \rho_{1} \in I$, we get that $\mu_{2} \beta_{s} \notin I$, so $\beta_{s} \ldots \beta_{1}$ is not the longest string starting from $i-1$, a contradiction. Similarly, we can prove that $N_{2}$ is injective. Therefore, inj. $\operatorname{dim} P_{i-1} \leq 1$.

If the indecomposable projective module $P$ satisfies that its corresponding vertex $i-1$ does not lie at any cycle $c \in \mathcal{C}(\Lambda)$, we assume that the quiver $Q$ looks as the following diagram shows. Note that there are at most one arrow $\alpha_{i}$ starting from $i-1$, and at most one arrow $\alpha_{i-1}$ ending to $i-1$.

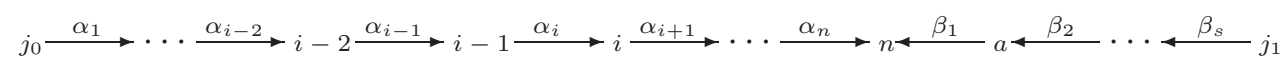

Figure 3. The quiver of $Q$.

Denote by $j_{0}$ the starting vertex of the longest path $\alpha_{i-1} \ldots \alpha_{1}$, and $n$ the ending vertex of the longest path $\alpha_{n} \ldots \alpha_{i}$. Also $j_{1}$ is the starting vertex of the longest path $\beta_{1} \ldots \beta_{s}$ ending to $n$. So $P_{i-1}$ is the string module with string

$$
i-1 \stackrel{\alpha_{i}}{\longrightarrow} i \stackrel{\alpha_{i+1}}{\longrightarrow} \ldots \stackrel{\alpha_{n}}{\longrightarrow} n .
$$

$I_{n}$ is the string module with string as Figure 3 shows. So there is a short exact sequence

$$
0 \rightarrow P_{i-1} \rightarrow I_{n} \rightarrow L_{1} \oplus L_{2} \rightarrow 0,
$$

where $L_{1}$ is the string module with string

$$
j_{0} \stackrel{\alpha_{1}}{\longrightarrow} \cdots \stackrel{\alpha_{i-2}}{\longrightarrow} i-2,
$$

and $L_{2}$ is the string module with string

$$
j_{1} \stackrel{\beta_{s}}{\longrightarrow} \cdots \stackrel{\beta_{2}}{\longrightarrow} a .
$$

We claim that $L_{1}$ and $L_{2}$ are injective modules. If $L_{2}$ is nonzero and not injective, then there exists one arrow $\gamma_{1}$ ending to $a$, which implies that $\beta_{1} \gamma_{1} \in I$ since $\beta_{1} \beta_{2} \notin I$. By the assumption, we know that there exists one arrow $\gamma_{2}$ starting from $n$ such that $\gamma_{2} \beta_{1} \in I$, then $\gamma_{2} \alpha_{n} \notin I$, contradicts to $\alpha_{n} \ldots \alpha_{i}$ is the longest path starting from $i-1$. So $L_{2}$ is injective. Similarly, we can prove that $L_{1}$ is injective. So we get that inj. $\operatorname{dim} P_{i-1} \leq 1$.

To sum up, for any indecomposable projective module $P$, we get that inj. $\operatorname{dim} P \leq 1$, which yields that $\operatorname{inj} . \operatorname{dim} \Lambda \leq 1$. Since $\Lambda$ is Gorenstein, we get that $\Lambda$ is 1 -Gorenstein. 
From now on, we only consider gentle algebras $\Lambda$ which is 1-Gorenstein.

For any gentle algebra $\Lambda$, from [23, we know that $\Lambda$ is CM-finite, and the Cohen-Macaulay Auslander algebra $\operatorname{Aus}(\operatorname{Gproj} \Lambda)=K Q^{A u s} /\left\langle I^{A u s}\right\rangle$ is the algebra with the bound quiver $\left(Q^{\text {Aus }}, I^{\text {Aus }}\right)$ defined as follows:

- the set of vertices $Q_{0}^{\text {Aus }}:=Q_{0} \bigsqcup Q_{1}^{c y c}$, where $Q_{1}^{c y c}=\{\alpha \mid \alpha \in \mathcal{C}(\Lambda)\}$;

- the set of arrows $Q_{1}^{\text {Aus }}:=Q_{1}^{n c y c} \bigsqcup\left(Q_{1}^{c y c}\right)^{ \pm}$, where $Q_{1}^{\text {ncyc }}=Q_{1} \backslash Q_{1}^{\text {cyc }}$, i.e. arrows do not appear in any cyclic paths in $\mathcal{C}(\Lambda),\left(Q_{1}^{c y c}\right)^{+}=\left\{\alpha^{+}: s(\alpha) \rightarrow \alpha \mid \alpha \in Q_{1}^{c y c}\right\}$ and $\left(Q_{1}^{c y c}\right)^{-}=$ $\left\{\alpha^{-}: \alpha \rightarrow t(\alpha) \mid \alpha \in Q_{1}^{c y c}\right\}$.

The ideal $I^{\text {Aus }}:=\left\{\beta^{+} \alpha^{-} \mid \beta \alpha \in I, \alpha, \beta \in Q_{1}^{c y c}\right\} \bigcup\left\{\beta \alpha \mid \beta \alpha \in I, \alpha, \beta \in Q_{1}^{\text {ncyc }}\right\}$.

Before going on, let us fix some notations. Let $\Lambda$ be a gentle algebra and $\Gamma$ be its CohenMacaulay Auslander algebra.

For any $M=\left(\left(M_{i}\right)_{i \in Q_{0}},\left(M_{\alpha}: M_{i} \rightarrow M_{j}\right)_{(\alpha: i \rightarrow j) \in Q_{1}}\right) \in \bmod \Lambda$, Define a $\Gamma$-module $\widehat{M}=$ $\left(\left(N_{i}, N_{\alpha}\right)_{i \in Q_{0}, \alpha \in Q_{1}^{c y c},}\left(N_{\beta}\right)_{\beta \in Q_{1}^{A u s}}\right)$ as follows:

- For any $i \in Q_{0} \subseteq Q_{0}^{\text {Aus }}$, we set $N_{i}=M_{i}$; For any $\alpha \in Q_{1}^{c y c} \subseteq Q_{1}^{A u s}$, we set $N_{\alpha}=\operatorname{Im} M_{\alpha}$.

- For any arrow in $Q_{1}^{\text {Aus }}$, if it is of form $(\beta: i \rightarrow j) \in Q_{1}^{n c y c}$, then we set $N_{\beta}=M_{\beta}$; if it is of form $\beta^{+}: i \rightarrow \beta$, or of form $\beta^{-}: \beta \rightarrow j$ for some $(\beta: i \rightarrow j) \in Q_{1}^{c y c}$, we set $N_{\beta^{+}}$and $N_{\beta^{-}}$ to be the natural morphisms $\left(N_{i}=M_{i}\right) \rightarrow\left(\operatorname{Im} M_{\beta}=N_{\beta}\right)$ and $\left(N_{\beta}=\operatorname{Im} M_{\beta}\right) \rightarrow\left(M_{j}=N_{j}\right)$ respectively induced by $M_{\beta}: M_{i} \rightarrow M_{j}$.

It is easy to see that $\widehat{M}$ is actually a $\Gamma$-module. Since $\operatorname{Im}$ is a functor, we can define a functor $\Phi: \bmod \Lambda \rightarrow \bmod \Gamma$ such that $\Phi(M):=\widehat{M}$, with the natural definition on morphisms.

Since $(Q, I)$ is a subquiver of $\left(Q^{A u s}, I^{A u s}\right)$, i.e. $\Lambda$ is a subalgebra of $\Gamma$, we get a restriction

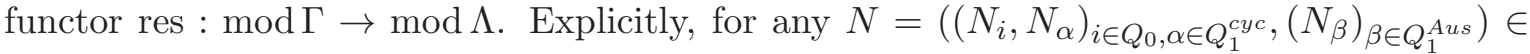
$\bmod \Gamma, \operatorname{res}(N)$ is defined as follows:

- For any $i \in Q_{0},(\operatorname{res}(N))_{i}=N_{i}$;

- For any arrow $(\alpha: i \rightarrow j) \in Q_{1}$, if $\alpha \in Q_{1}^{n c y c}$, we set $(\operatorname{res} N)_{\alpha}=N_{\alpha}$; if $\alpha \in Q_{1}^{c y c}$, we set $(\operatorname{res}(N))_{\alpha}=N_{\alpha^{-}} N_{\alpha^{+}}$.

Lemma 3.2. We have $\operatorname{res}(\Phi(M)) \simeq M$ naturally.

Proof. This follows from the definition of the functors $\Phi$ and res immediately.

Similar to [20, Lemma 5.3], we also have the following weak adjunction properties:

Lemma 3.3. (i) For all $M \in \bmod \Lambda$ and $N \in \bmod \Gamma$, the natural maps

$$
\operatorname{Hom}_{\Gamma}(\widehat{M}, N) \stackrel{\text { res }}{\longrightarrow} \operatorname{Hom}_{\Lambda}(\operatorname{res} \widehat{M}, \operatorname{res} N) \simeq \operatorname{Hom}_{\Lambda}(M, \operatorname{res} N)
$$

and

are injective.

$$
\operatorname{Hom}_{\Gamma}(N, \widehat{M}) \stackrel{\text { res }}{\longrightarrow} \operatorname{Hom}_{\Lambda}(\operatorname{res} N, \operatorname{res} \widehat{M}) \simeq \operatorname{Hom}_{\Lambda}(\operatorname{res} N, M)
$$

(ii) The functor $\Phi$ is fully faithful. In particular, $\Phi$ preserves injective and surjective morphisms.

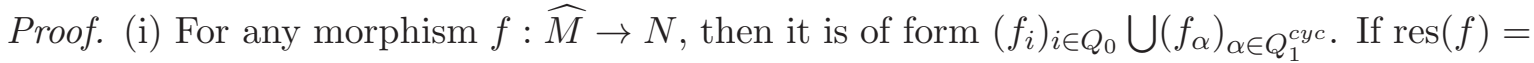
0 , then $\operatorname{res}(f)=\left(f_{i}\right)_{i \in Q_{0}}=0$. Let $M=\left(\left(M_{i}\right)_{i \in Q_{0}},\left(M_{\alpha}\right)_{(\alpha: i \rightarrow j) \in Q_{1}}\right)$ and $N=\left(\left(N_{i}\right)_{i \in Q_{0}} \cup\right.$

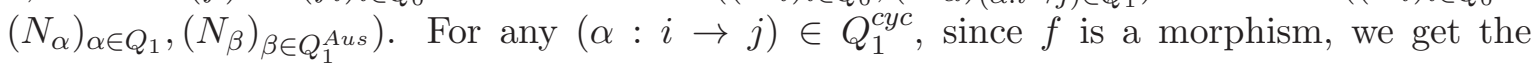
following commutative diagram

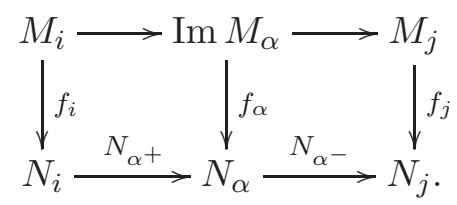


Since $f_{i}=f_{j}=0$ and the first map in the upper row is surjective, we get that $f_{\alpha}=0$. So $\operatorname{Hom}_{\Gamma}(\widehat{M}, N) \stackrel{\text { res }}{\longrightarrow} \operatorname{Hom}_{\Lambda}($ res $\widehat{M}$, res $N)$ is injective. The second statement can be proved dually.

(ii) For all $M, N$ in $\bmod \Lambda$, we have a chain of maps

$$
\operatorname{Hom}_{\Lambda}(M, N) \stackrel{\Phi}{\rightarrow} \operatorname{Hom}_{\Gamma}(\widehat{M}, \widehat{N}) \stackrel{\text { res }}{\longrightarrow} \operatorname{Hom}_{\Lambda}(\operatorname{res} \widehat{M}, \operatorname{res} \widehat{N}) \simeq \operatorname{Hom}_{\Lambda}(M, N),
$$

whose composition is the identity, thus the first map is injective. The second map is injective due to (i). So $\Phi$ is fully faithful.

Since $\Lambda$ and $\Gamma$ are gentle algebras, their indecomposable modules are either string modules or band modules. We describe the action of $\Phi$ and res on string modules as follows.

- For a string $w=\alpha_{1}^{\epsilon_{1}} \alpha_{2}^{\epsilon_{2}} \ldots \alpha_{n}^{\epsilon_{n}} \in \mathcal{S}(\Lambda)$, denote the string module by $M(w)$. For $i=$ $1, \ldots, n$, if $\alpha_{i} \in Q_{1}^{c y c}$, we replace $\alpha_{i}$ by $\alpha_{i}^{-} \alpha_{i}^{+}$, and get a word in $\Gamma$, denote it by $\iota(w)$. Then it is easy to see that $\iota(w) \in \mathcal{S}(\Gamma)$, we denote its string module by $N(\iota(w))$. Note that

$$
\underline{\operatorname{dim}} N(\iota(w))=\underline{\operatorname{dim}} M(w)+\sum_{\alpha_{i} \in Q_{1}^{c y c}} \underline{\operatorname{dim}} S_{\alpha_{i}},
$$

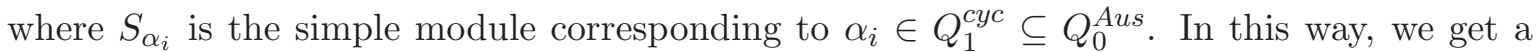
map $\iota: \mathcal{S}(\Lambda) \rightarrow \mathcal{S}(\Gamma)$, which is injective. It is easy to see that $\Phi(M(w))=N(\iota(w))$.

- For a string $v=\beta_{1} \beta_{2} \ldots \beta_{n} \in \mathcal{S}(\Gamma)$, denote the string module by $N(v)$. Denote by $v^{\prime}$ the longest substring of $v$ such that $s\left(v^{\prime}\right), t\left(v^{\prime}\right) \in Q_{0} \subseteq Q_{0}^{\text {Aus }}$. Note that $l(v)-l\left(v^{\prime}\right) \leq 2$ and $l\left(v^{\prime \prime}\right)-l(v) \leq 2$. If $\alpha^{-} \alpha^{+}$(or its inverse) appears as a subword of $v^{\prime}$ for any arrow $\alpha \in Q_{1}^{c y c}$, we replace $\alpha^{-} \alpha^{+}$(or its inverse) by $\alpha$ (or $\alpha^{-1}$ ), after doing this repeatedly, finally we can get a word in $\Lambda$, denote it by $\pi^{-}(v)$. Then it is easy to see that $\pi^{-}(v) \in \mathcal{S}(\Lambda)$, we denote its string module by $M\left(\pi^{-}(v)\right)$. Note that if $\operatorname{dim} N(v)=\left(k_{i}\right)_{i \in Q_{0}}+\left(k_{\alpha}\right)_{\alpha \in Q_{1}^{c y c}}$, then $\operatorname{dim} M\left(\pi^{-}(v)\right)=\left(k_{i}\right)_{i \in Q_{0}}$. In this way, we get a surjective map $\pi^{-}: \mathcal{S}(\Gamma) \rightarrow \mathcal{S}(\Lambda)$, in fact, $\pi^{-} \iota=$ Id. Easily, $\operatorname{res}(N(v))=M\left(\pi^{-}(v)\right)$.

Lemma 3.4. Let $\Lambda$ be a gentle algebra, and $\Gamma$ be its Cohen-Macaulay Auslander algebra. Then for any projective $\Lambda$-module $P$, and any injective $\Lambda$-module $I$, we have $\Phi(P)$ is a projective $\Gamma$-module, and $\Phi(I)$ is an injective $\Gamma$-module. In other words, $\Phi$ preserves projectives and injectives.

Proof. From [34, we get that the indecomposable projective $\Lambda$-modules $P$ are of the following form:

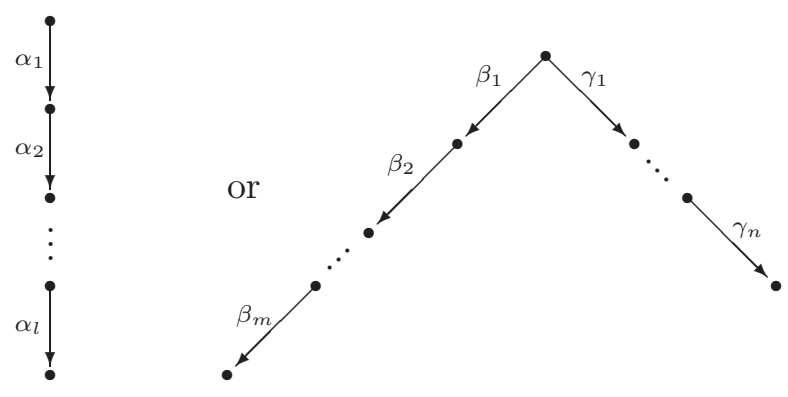

Figure 3. The strings of projective modules.

They correspond to the strings $\alpha_{l} \ldots \alpha_{1}$ and $\beta_{m} \ldots \beta_{1} \gamma_{1}^{-1} \ldots \gamma_{n}^{-1}$, respectively. In particular, $\alpha_{l} \ldots \alpha_{1}, \beta_{m} \ldots \beta_{1}$ and $\gamma_{n} \ldots \gamma_{1}$ are maximal, e.g. there does not exist $\alpha \in Q_{1}$ such that $\alpha \alpha_{l} \notin I$. 
In the first case, $\iota\left(\alpha_{l} \ldots \alpha_{1}\right) \in \mathcal{S}(\Lambda)$ is also maximal. In fact, if there exists an arrow in $Q_{1}^{\text {Aus }}$, which is either of form $\xi \in Q_{1} \subseteq Q_{1}^{\text {Aus }}$ or of form $\xi^{+}$for some $\xi \in Q_{1}$, such that $\xi \iota\left(\alpha_{l} \ldots \alpha_{1}\right)$ or $\xi^{+} \iota\left(\alpha_{l} \ldots \alpha_{1}\right)$ is also a string, then $\xi \alpha_{l} \notin I$ in both cases, so $\xi \alpha_{l} \ldots \alpha_{1}$ is a string in $\mathcal{S}(\Lambda)$, a contradiction.

Note that we can view $s\left(\alpha_{1}\right)$ to be a vertex in $Q_{0}^{A u s}$. From the structure of $Q^{A u s}$, there is no other arrow than $\alpha_{1}$ (if $\alpha_{1} \in Q_{1}^{n c y c}$ ) or $\alpha_{1}^{+}$(if $\alpha_{1} \in Q_{1}^{c y c}$ ) starting from $s\left(\alpha_{1}\right)$. So $\iota\left(\alpha_{l} \ldots \alpha_{1}\right)$ is the string of the indecomposable projective $\Gamma$-module corresponding to the vertex $s\left(\alpha_{1}\right)$ for $\Gamma$ is gentle.

The second case is similar to the first one, we omit the proof here.

For $\Phi$ preserves injectives, it is dual to the above, we omit the proof here.

Lemma 3.5. Let $\Lambda$ be a 1-Gorenstein gentle algebra, and $\Gamma$ be its Cohen-Macaulay Auslander algebra. Then for any $M \in G \operatorname{Groj} \Lambda$, proj. $\operatorname{dim} \Phi(M) \leq 1$ and inj. $\operatorname{dim} \Phi(M) \leq 1$.

Proof. We only prove it for the case when $M$ is an indecomposable Gorenstein projective module.

Case (1). If $M$ is projective, then Lemma 3.4 yields that $\Phi(M)$ is also projective. We denote by $i-1$ the vertex corresponding to the indecomposable projective module $M$.

Case (1a). If the corresponding vertex $i-1$ lies at a cycle $c=\alpha_{n} \ldots \alpha_{1} \in \mathcal{C}(\Lambda)$, we assume the quiver $Q$ looks as Figure 2 shows. Then there is short exact sequence:

$$
0 \rightarrow P_{i-1} \stackrel{f}{\rightarrow} I_{j_{2}} \oplus I_{j_{3}} \stackrel{g}{\rightarrow} I_{i-1} \oplus I_{a} \oplus I_{b} \rightarrow 0,
$$

Lemma 3.3 implies $\Phi(f)$ is also injective. We denote by $U_{1}$ (resp. $U_{2}$ ) the indecomposable $\Gamma$-module corresponding to the string $\iota\left(\rho_{2} \ldots \rho_{v}\right)$ (resp. $\left.\iota\left(\delta_{2} \ldots \delta_{u}\right)\right)$ if $\rho_{1} \in Q_{1}^{n c y c}$ (resp. $\delta_{1} \in$ $\left.Q_{1}^{n c y c}\right)$, and to the string $\rho_{1}^{+} \iota\left(\rho_{2} \ldots \rho_{v}\right)\left(\right.$ resp. $\left.\delta_{1}^{+} \iota\left(\delta_{2} \ldots \delta_{u}\right)\right)$ if $\rho_{1} \in Q_{1}^{c y c}\left(\operatorname{resp} . \delta \in Q_{1}^{c y c}\right)$.

We claim that $U_{1}$ and $U_{2}$ are injective. We only prove it for $U_{1}$. If $\rho_{1} \in Q_{1}^{\text {ncyc }}$, then it follows from Lemma 3.4 that $U_{1}$ is injective. Otherwise, if $\rho_{1} \in Q_{1}^{c y c}$, then $\rho_{1}^{+} \iota\left(\rho_{2} \ldots \rho_{v}\right)$ is a string with ending vertex $\rho_{1} \in Q_{1}^{\text {Aus }}$, and $\iota\left(\rho_{2} \ldots \rho_{v}\right)$ is the string of an indecomposable injective module, which implies $\iota\left(\rho_{2} \ldots \rho_{v}\right)$ is maximal. Note that there is only one arrow $\rho_{1}^{+}$ with ending vertex $\rho_{1}$. So $\rho_{1}^{+} \iota\left(\rho_{2} \ldots \rho_{v}\right)$ is the string of the indecomposable injective module corresponding to vertex $\rho_{1} \in Q_{1}^{\text {Aus }}$.

By the above construction, it is easy to see that the following sequence is an injective resolution of $\Phi(M)=\Phi\left(P_{i-1}\right)$ :

$$
0 \rightarrow \Phi\left(P_{i-1}\right) \stackrel{\Phi(f)}{\longrightarrow} \Phi\left(I_{j_{2}}\right) \oplus \Phi\left(I_{j_{3}}\right) \rightarrow \Phi\left(I_{i-1}\right) \oplus U_{1} \oplus U_{2} \rightarrow 0,
$$

since $\Phi$ preserves injectives. So inj. $\operatorname{dim} \Phi\left(P_{i-1}\right) \leq 1$.

Case (1b). If the corresponding vertex $i-1$ does not lie at any cycle in $\mathcal{C}(\Lambda)$, we assume that the quiver $Q$ looks as Figure 3 shows. The remaining is similar to Case (1a), we omit the proof here.

Case (2). If $M=R\left(\alpha_{i-1}\right)$ for some $\alpha_{i-1}$ lying on some cycle $c=\alpha_{n} \ldots \alpha_{1} \in \mathcal{C}(\Lambda)$, we assume that the quiver $Q$ looks as Figure 2 shows. Then $R\left(\alpha_{i-1}\right)$ is the string module with its string $\beta_{s} \ldots \beta_{1}$. There is a short exact sequence

$$
0 \rightarrow R\left(\alpha_{i}\right) \stackrel{f}{\rightarrow} P_{i-1} \stackrel{g}{\rightarrow} R\left(\alpha_{i-1}\right) \rightarrow 0 .
$$

So $\Phi\left(R\left(\alpha_{i-1}\right)\right)$ is the $\Gamma$-module corresponding to the string $\iota\left(\beta_{s} \ldots \beta_{1}\right)$. Denote by $U_{\alpha_{i}}$ the $\Gamma$-module corresponding to the string $\iota\left(\gamma_{t} \ldots \gamma_{1}\right) \alpha_{i}^{-} \in \mathcal{C}(\Gamma)$. Since there is only one arrow $\alpha_{i}^{-}$starting from $\alpha_{i} \in Q_{0}^{\text {Aus }}$, and $\gamma_{t} \ldots \gamma_{1}$ is maximal, we get that $U_{\alpha_{i}}$ is the indecomposable projective $\Gamma$-module corresponding to the vertex $\alpha_{i}$. From this construction, it is easy to see 
that the following sequence is a projective resolution of $\Phi\left(R\left(\alpha_{i-1}\right)\right)$ :

$$
0 \rightarrow U_{\alpha_{i}} \rightarrow \Phi\left(P_{i-1}\right) \stackrel{\Phi(g)}{\longrightarrow} \Phi\left(R\left(\alpha_{i-1}\right)\right) \rightarrow 0,
$$

since $\Phi$ preserves projectives and epimorphisms. So proj. $\operatorname{dim} \Phi\left(R\left(\alpha_{i-1}\right)\right) \leq 1$.

We denote by $J_{\alpha_{i-1}}$ the indecomposable injective $\Gamma$-module corresponding to the vertex $\alpha_{i-1} \in Q_{1}^{\text {Aus }}$, also $U_{1}$ the indecomposable $\Gamma$-module corresponding to the string $\iota\left(\rho_{2} \ldots \rho_{v}\right)$ if $\rho_{1} \in Q_{1}^{\text {ncyc }}$, and to the string $\rho_{1}^{+} \iota\left(\rho_{2} \ldots \rho_{v}\right)$ if $\rho_{1} \in Q_{1}^{c y c}$. By the above, $U_{1}$ is also injective. Then the following sequence is an injective resolution of $\Phi\left(R\left(\alpha_{i-1}\right)\right)$ :

$$
0 \rightarrow \Phi\left(R\left(\alpha_{i-1}\right)\right) \rightarrow \Phi\left(I_{j_{2}}\right) \rightarrow J_{\alpha_{i-1}} \oplus U_{1} \rightarrow 0 .
$$

So inj. $\operatorname{dim} \Phi\left(R\left(\alpha_{i-1}\right)\right) \leq 1$.

In conclusion, for any $M \in \operatorname{Gproj} \Lambda$, we have that proj. $\operatorname{dim} \Phi(M) \leq 1$ and inj. $\operatorname{dim} \Phi(M) \leq$ 1 .

Lemma 3.6. Let $\Lambda$ be a 1-Gorenstein gentle algebra, and $\Gamma$ be its Cohen-Macaulay Auslander algebra. Then for any $M \in \operatorname{Gproj} \Lambda, \operatorname{Ext}_{\Gamma}^{1}(\Phi(M), \Phi(M))=0$.

Proof. We only need prove that for any two indecomposable Gorenstein projective $\Lambda$-modules $M_{1}, M_{2}, \operatorname{Ext}_{\Gamma}^{1}\left(\Phi\left(M_{1}\right), \Phi\left(M_{2}\right)\right)=0$.

If $M_{1}$ is projective, then $\Phi\left(M_{1}\right)$ is also projective, so $\operatorname{Ext}_{\Gamma}^{1}\left(\Phi\left(M_{1}\right), \Phi\left(M_{2}\right)\right)=0$ for any $M_{2} \in \operatorname{Gproj} \Lambda$. For this, we assume that $M_{1}=R\left(\alpha_{i-1}\right)$ for some $\alpha_{i-1} \in Q_{1}^{c y c}$. We also assume the quiver $Q$ looks as Figure 2 shows. Then there is a short exact sequence:

$$
0 \rightarrow U_{\alpha_{i}} \stackrel{f}{\rightarrow} \Phi\left(P_{i-1}\right) \stackrel{\Phi(g)}{\longrightarrow} \Phi\left(R\left(\alpha_{i-1}\right)\right) \rightarrow 0,
$$

where $U_{\alpha_{i}}$ is the $\Gamma$-module corresponding to the string $\iota\left(\gamma_{t} \ldots \gamma_{1}\right) \alpha_{i}^{-} \in \mathcal{S}(\Gamma)$, which is the indecomposable projective module corresponding to the vertex $\alpha_{i} \in Q_{0}^{\text {Aus }} ; \Phi\left(P_{i-1}\right)$ is the indecomposable projective $\Gamma$-module corresponding to the vertex $i-1 \in Q_{0}^{\text {Aus }}$. Note that $f$ is induced by $\left(\alpha_{i}^{+}: i-1 \rightarrow \alpha_{i}\right) \in Q_{1}^{\text {Aus }}$.

If $M_{2}$ is projective, which is of form $P_{k}$ corresponding to the vertex $k$, Then $\Phi\left(M_{2}\right)=\Phi\left(P_{k}\right)$ is also projective. For any nonzero morphism $h: U_{\alpha_{i}} \rightarrow \Phi\left(P_{k}\right)$, we can assume that $h$ is induced by a nonzero path $l$ from $k$ to $\alpha_{i}$ in $\Gamma$. Since $k \in Q_{0} \subseteq Q_{0}^{\text {Aus }}$ and $k \neq \alpha_{i}$, the length of $l$ is not zero. There is only one arrow $\alpha_{i}^{+}$ending to $\alpha_{i} \in Q_{1}^{\text {Aus }}$, so $l=\alpha_{i}^{+} l^{\prime}$ for some nonzero path $l^{\prime}$, which implies that $h$ factors through $f$. So we get that

$$
\operatorname{Hom}_{\Gamma}\left(\Phi\left(P_{i-1}\right), \Phi\left(P_{k}\right)\right) \stackrel{\operatorname{Hom}_{\Gamma}\left(f, \Phi\left(P_{k}\right)\right)}{\longrightarrow} \operatorname{Hom}_{\Gamma}\left(U_{\alpha_{i}}, \Phi\left(P_{k}\right)\right)
$$

is surjective. By applying $\operatorname{Hom}_{\Gamma}\left(-, \Phi\left(P_{k}\right)\right)$ to Sequence (2), we get that

$$
\operatorname{Ext}_{\Gamma}^{1}\left(\Phi\left(R\left(\alpha_{i-1}\right)\right), \Phi\left(P_{k}\right)\right)=0 .
$$

If $M_{2}=R\left(\beta_{j-1}\right)$, where $\beta_{j-1}$ lies on a cycle $\beta_{m} \ldots \beta_{1} \in \mathcal{C}(\Lambda)$, we denote by $\sigma_{k} \ldots \sigma_{1}$ be the string of $R\left(\beta_{j-1}\right)$ :

$$
j-1 \stackrel{\sigma_{1}}{\longrightarrow} \bullet \stackrel{\sigma_{2}}{\longrightarrow} \cdots \stackrel{\sigma_{k}}{\longrightarrow} \bullet .
$$

For any nonzero morphism $p: U_{\alpha_{i}} \rightarrow \Phi\left(R\left(\beta_{j-1}\right)\right)$, since $U_{\alpha_{i}}$ is the indecomposable projective module corresponding to the vertex $\alpha_{i}$, we assume that $p$ is induced by a nonzero path $l$ from $j-1$ to $\alpha_{i}$. Since there is only one arrow $\alpha_{i}^{+}$ending to $\alpha_{i}$ and $j-1 \in Q_{0} \subseteq Q_{0}^{\text {Aus }}$, we get that $l=l^{\prime} \alpha_{i}^{+}$. Note that $f$ is induced by $\alpha_{i}^{+}$. We get that $p$ factors through $f$ as $p=p^{\prime} f$ for some morphism $p^{\prime}: \Phi\left(P_{i-1}\right) \rightarrow \Phi\left(R\left(\beta_{j-1}\right)\right)$. Therefore,

$$
\operatorname{Hom}_{\Gamma}\left(\Phi\left(P_{i-1}\right), \Phi\left(R\left(\beta_{j-1}\right)\right)\right) \stackrel{\operatorname{Hom}_{\Gamma}\left(f, \Phi\left(R\left(\beta_{j-1}\right)\right)\right)}{\longrightarrow} \operatorname{Hom}_{\Gamma}\left(U_{\alpha_{i}}, \Phi\left(R\left(\beta_{j-1}\right)\right)\right)
$$


is surjective. By applying $\operatorname{Hom}_{\Gamma}\left(-, \Phi\left(P_{k}\right)\right)$ to Sequence (2), we get that

$$
\operatorname{Ext}_{\Gamma}^{1}\left(\Phi\left(R\left(\alpha_{i-1}\right)\right), \Phi\left(R\left(\beta_{j-1}\right)\right)\right)=0 .
$$

In conclusion, we get that $\operatorname{Ext}_{\Gamma}^{1}\left(\Phi\left(M_{1}\right), \Phi\left(M_{2}\right)\right)=0$ for any two indecomposable Gorenstein projective $\Lambda$-modules $M_{1}, M_{2}$. So for any $M \in \operatorname{Gproj} \Lambda, \operatorname{Ext}_{\Gamma}^{1}(\Phi(M), \Phi(M))=0$.

Theorem 3.7. Let $\Lambda=K Q /\langle I\rangle$ be a gentle algebra which is 1-Gorenstein, and $\Gamma=$ $K Q^{\text {Aus }} /\left\langle I^{\text {Aus }}\right\rangle$ its Cohen-Macaulay Auslander algebra. Let $M$ be a Gorenstein projective $\Lambda$-module and let e be a dimension vector for $Q^{\text {Aus }}$. Then

(i) $\Gamma$ has global dimension at most two, both the injective and the projective dimension of $\Phi(M)$ over $\Gamma$ are at most one, and $\operatorname{Ext}_{\Gamma}^{1}(\Phi(M), \Phi(M))=0$;

(ii) The quiver Grassmannian $\mathrm{Gr}_{e}(\Phi(M))$ is smooth (and reduced), with irreducible and equidimensional connected components.

Proof. It follows from Lemma 3.5, Lemma 3.6 and Lemma 2.9 immediately.

\section{Construction of the Desingularizations}

This section is based on [20, Section 7]. In this section, we always assume that $\Lambda=K Q /\langle I\rangle$ to be a 1-Gorenstein gentle algebra, and $\Gamma$ be its Cohen-Macaulay Auslander algebra. For a given Gorenstein projective $\Lambda$-module $M$, a dimension vector $e$ and an isomorphism classes $[N]$ of $\Lambda$-modules, it is proved in [18, Section 2.3] that the subset $\mathcal{S}_{[N]}$ of $\mathrm{Gr}_{e}(M)$, consisting of the submodules which are isomorphic to $N$, is locally closed and irreducible of dimension $\operatorname{dim} \operatorname{Hom}_{\Lambda}(N, M)-\operatorname{dim} \operatorname{End}_{\Lambda}(N)$.

Lemma 4.1 (see e.g. [21]). If $\Lambda$ is 1-Gorenstein, then the Gorenstein projective modules are just torsionless modules. In particular, for any submodule $N$ of any Gorenstein projective $\Lambda$-module $M, N$ is also Gorenstein projective.

Lemma 4.2. For any Gorenstein projective $\Lambda=K Q /\langle I\rangle$-module $N=\left(\left(N_{i}\right)_{i \in Q_{0}},\left(N_{\alpha}\right)_{\alpha \in Q_{1}}\right)$, the underlying space of $\widehat{N}$ is

$$
\bigoplus_{i \in Q_{0}} \widehat{N}_{i} \oplus \bigoplus_{\alpha \in Q_{1}^{c y c}} \widehat{N}_{\alpha}
$$

Then

(i) $\operatorname{dim} \widehat{N}_{i}=\operatorname{dim} N_{i}=\operatorname{dim} \operatorname{Hom}_{\Lambda}\left(P_{i}, N\right)$, where $P_{i}$ is the indecomposable projective module corresponding to $i$.

(ii) $\operatorname{dim} \widehat{N}_{\alpha}=\operatorname{dim} \operatorname{Hom}_{\Lambda}\left(P_{s(\alpha)}, N\right)-\operatorname{dim} \operatorname{Hom}_{\Lambda}(R(\beta), N)$, where $P_{s(\alpha)}$ is the indecomposable projective module corresponding to $s(\alpha)$, and $\beta$ is the arrow satisfying $\alpha \beta \in I$.

Proof. Without losing generality, we assume that $N$ is indecomposable.

(i) is obvious from the definition of $\Phi$. For (ii), by Sequence (1), there is an exact sequence

$$
0 \rightarrow R(\alpha) \stackrel{f}{\rightarrow} P_{s(\alpha)} \stackrel{g}{\rightarrow} R(\beta) \rightarrow 0 .
$$

Note that $N$ is a string module. Let $w$ be the string of $N$. By the definition of $\Phi$ and $\iota$, it is easy to see that $\operatorname{dim} \widehat{N}_{\alpha}$ is equal to the times of $w$ passing through $\alpha$. Let $v$ be the string of $R(\beta)$. Then the quiver of $Q$ locally looks like as the following.

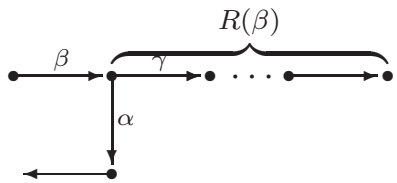


Note that $v$ is maximal and $\operatorname{Hom}_{\Lambda}\left(P_{s(\alpha)}, N\right)$ is equal to the times of $w$ passing through $s(\alpha)$. We get that $\operatorname{dim} \operatorname{Hom}_{\Lambda}(R(\beta), N)$ is equal to the times of $w$ passing through $\gamma$ if the length of $v$ is not zero, or $s(\alpha)$ is an ending point of $v$ if the length of $v$ is zero. So the times of $w$ passing through $\alpha$ is equal to $\operatorname{dim}_{\operatorname{Hom}_{\Lambda}}\left(P_{s(\alpha)}, N\right)-\operatorname{dim}_{\operatorname{Hom}}(R(\beta), N)$, which is also equal to $\operatorname{dim} \widehat{N}_{\alpha}$ for any $\alpha \in Q_{1}^{c y c}$.

Proposition 4.3. Suppose that $\mathcal{S}_{[U]}$ has non-empty intersection with $\overline{\mathcal{S}_{[N]}}$. Then $\underline{\operatorname{dim}} \widehat{U} \leq$ dim $\widehat{N}$ componentwise as dimension vectors of representations of $\Gamma$.

Proof. Following [18, Section 2.3], we write

$$
\operatorname{Hom}^{0}(e, M):=\{(N, f) \mid N \in \bmod \Lambda, \underline{\operatorname{dim}} N=e \text { and } f: N \rightarrow M \text { is injective }\} .
$$

Then $\operatorname{Gr}_{e}(M)$ is isomorphic to the quotient $\operatorname{Hom}^{0}(e, M) / G_{e}$. We have a canonical map

$$
p: \operatorname{Hom}^{0}(e, M) \rightarrow R_{e}(\Lambda)
$$

which maps $(N, f)$ to $N$. We also denote by $\pi: \operatorname{Hom}^{0}(e, M) \rightarrow \operatorname{Gr}_{e}(M)$ the locally trivial $G_{e}$-principle bundle. Then the stratum $\mathcal{S}_{[N]}$ is then defined by $\pi\left(p^{-1}\left(\mathcal{O}_{[N]}\right)\right)$.

Similar to [20, Proposition 7.2], we know that if $\mathcal{S}_{[U]}$ has non-empty intersection with $\overline{\mathcal{S}_{[N]}}$, then $\mathcal{O}_{[U]} \subset \overline{\mathcal{O}_{[N]}}$. So $\operatorname{dim} \operatorname{Hom}_{\Lambda}(P, U)=\operatorname{dim} \operatorname{Hom}_{\Lambda}(P, N)$ for all projective representations $P$, and $\operatorname{dim} \operatorname{Hom}_{\Lambda}(X, U) \geq \operatorname{dim} \operatorname{Hom}_{\Lambda}(X, N)$ for all non-projectives $X$ (see 10]). Note that $\operatorname{dim} U_{i}=\operatorname{dim} N_{i}$ for any $i \in Q_{0}$. Now consider the dimension vector of $\widehat{U}$, resp. of $\widehat{N}$. Then Lemma 4.2 (i) implies that $\operatorname{dim} \widehat{U}_{i}=\operatorname{dim} U_{i}=\operatorname{dim} N_{i}=\operatorname{dim} \widehat{N}_{i}$ for any $i \in Q_{0} \subseteq Q_{0}^{\text {Aus }}$. For any $\alpha \in Q_{1}^{\text {cyc }} \subset Q_{1}^{\text {Aus }}$, there exists an arrow $\beta$ such that $\alpha \beta \in I$. Lemma 4.2 (ii) yields that

$$
\begin{aligned}
\operatorname{dim} \widehat{U}_{\alpha} & =\operatorname{dim} \operatorname{Hom}_{\Lambda}\left(P_{s(\alpha)}, U\right)-\operatorname{dim} \operatorname{Hom}_{\Lambda}(R(\beta), U) \\
& \leq \operatorname{dim} \operatorname{Hom}_{\Lambda}\left(P_{s(\alpha)}, N\right)-\operatorname{dim} \operatorname{Hom}_{\Lambda}(R(\beta), N),
\end{aligned}
$$

since $R(\beta)$ is not projective. This proves $\underline{\operatorname{dim}} \widehat{U} \leq \underline{\operatorname{dim}} \widehat{N}$ componentwise.

Definition $4.4([20])$. We call $[N]$ a generic subrepresentation type of $M$ of dimension vector $e$ if the stratum $\mathcal{S}_{[N]}$ of $\operatorname{Gr}_{e}(M)$ is open. Denote by $\operatorname{gsub}_{e}(M)$ the set of all generic subrepresentation types.

In case $[N] \in \operatorname{gsub}_{e}(M)$, the closure $\overline{\mathcal{S}_{[N]}}$ is an irreducible component of $\operatorname{Gr}_{e}(M)$, and every irreducible component arises in this way.

For any two Gorenstein projective $\Lambda$-modules $M$ and $N$, we consider quiver Grassmannians for $\Gamma$ of form $\operatorname{Gr}_{\underline{\underline{d i m}} \widehat{N}}(\widehat{M})$. For $[N] \in \operatorname{gsub}_{e}(M)$, we consider the map

$$
\pi_{[N]}: \operatorname{Gr}_{\underline{\underline{d i m}} \widehat{N}}(\widehat{M}) \rightarrow \operatorname{Gr}_{e}(M)
$$

given by $(F \subset \widehat{M}) \mapsto(\operatorname{res}(F) \subset M)$.

Proposition 4.5. For $M, e$ and $[N]$ as above and a point $(U \subset M)$ in $\operatorname{Gr}_{e}(M)$, we have an isomorphism

$$
\pi_{[N]}^{-1}(U \subset M) \cong \mathrm{Gr}_{\underline{\operatorname{dim}} \widehat{N}-\underline{\operatorname{dim}} \widehat{U}}(\widehat{M} / \widehat{U}) .
$$

Proof. More precisely, we prove that

$$
\pi_{[N]}^{-1}(U \subset M) \cong\{F \subset \widehat{M} \mid \underline{\operatorname{dim}} F=\underline{\operatorname{dim}} \widehat{N}, \widehat{U} \subset F\} .
$$

By definition of the map $\pi_{[N]}$, this immediately reduces to the following statement: Suppose we are given a subrepresentation $U \subset M$ of dimension vector $e$ and a subobject $F \subset \widehat{M}$ such that $\underline{\operatorname{dim}} F=\underline{\operatorname{dim}} \widehat{N}$. Then we have $\pi^{-}(F)=U$ if and only if $\widehat{U} \subset F$. 
So suppose $\underline{\operatorname{dim}} F=\underline{\operatorname{dim}} \widehat{N}$ and $\widehat{U} \subset F$. Then $U=\pi^{-}(\widehat{U}) \subset \pi^{-}(F)$ and

$$
\operatorname{dim} U_{i}=\operatorname{dim} \widehat{U}_{i}=\operatorname{dim} F_{i}=\operatorname{dim}\left(\pi^{-}(F)\right)_{i},
$$

for any $i \in Q_{0}$, and thus $U=\pi^{-}(F)$.

Conversely, suppose that $\pi^{-}(F)=U$ and $F \subset \widehat{M}$. Since $M$ is a Gorenstein projective module, $\pi^{-} \widehat{M}=M$, and $\pi^{-}$is exact, we get that $\pi^{-}(F) \subseteq M$, and then $\pi^{-}(F)$ is also a Gorenstein projective $\Lambda$-module. We denote by $F=\oplus F_{i}$, where $F_{i}$ are the indecomposable summands of $F . F \subset \widehat{M}$ implies that $F_{i}$ can be the simple $\Gamma$-module $S_{\alpha}$ for any $\alpha \in Q_{1}^{c y c}$. We denote by $w_{i}$ be the string of $F_{i}$. Then $w_{i}$ must be one of the following forms
(1) $\iota\left(\alpha_{l} \ldots \alpha_{1}\right)$;
(2) $\iota\left(\beta_{m} \ldots \beta_{1} \gamma_{1}^{-1} \ldots \gamma_{n}^{-1}\right)$;
(3) $\iota\left(\alpha_{l} \ldots \alpha_{1}\right) \alpha^{-}$;
(4) $\left(\beta^{-}\right)^{-1} \iota\left(\alpha_{l} \ldots \alpha_{1}\right)$;
(5) $\left(\beta^{-}\right)^{-1} \iota\left(\alpha_{l} \ldots \alpha_{1}\right) \alpha^{-}$;
(6) $\left(\alpha^{-}\right)^{-1} \iota\left(\beta_{m} \ldots \beta_{1} \gamma_{1}^{-1} \ldots \gamma_{n}^{-1}\right)$;
(7) $\iota\left(\beta_{m} \ldots \beta_{1} \gamma_{1}^{-1} \ldots \gamma_{n}^{-1}\right) \beta^{-}$;
(8) $\left(\alpha^{-}\right)^{-1} \iota\left(\beta_{m} \ldots \beta_{1} \gamma_{1}^{-1} \ldots \gamma_{n}^{-1}\right) \beta^{-}$,

since $\pi^{-}\left(F_{i}\right)$ is Gorenstein projective. From the above, we get that there exists a natural injective morphism $h: \Phi\left(\pi^{-}(F)\right) \rightarrow F$. In fact, $\pi^{-}$is exact, we get that $\pi^{-}(\operatorname{ker} h)=0$, since $\pi^{-}(h)=$ Id. Then ker $h \cong \bigoplus_{\alpha \in Q_{1}^{c y c}} S_{\alpha}^{n_{\alpha}}$. However, $S_{\alpha}$ can not be a submodule of $\Phi\left(\pi^{-}(F)\right)$, so ker $h=0$, and then $h$ is injective. So $\widehat{U}=\Phi\left(\pi^{-}(F)\right)$ is a submodule of $F$.

We can now easily derive the main general geometric properties of the map $\pi_{[N]}$ :

Theorem 4.6. For all $M$ and $e$ as above and a generic subrepresentation type $[N] \in$ $\operatorname{gsub}_{e}(M)$, the following holds:

(i) The varieties $\operatorname{Gr}_{\underline{\underline{\operatorname{dim}}} \widehat{N}}(\widehat{M})$ is smooth with irreducible equidimensional connected components;

(ii) the map $\pi_{[N]}$ is projective;

(iii) the image of $\pi_{[N]}$ is closed in $\operatorname{Gr}_{e}(M)$ and contains $\overline{\mathcal{S}_{[N]}}$;

(iv) the map $\pi_{[N]}$ is one-to-one over $\mathcal{S}_{[N]}$.

Proof. (i) follows from Theorem 3.7 directly. The map $\pi_{[N]}$ is projective since $\operatorname{Gr}_{\underline{\operatorname{dim}} \widehat{N}}(\widehat{M})$ is projective, so (ii) is valid.

For (iii), given a generic embedding $N \subset M$, we also have $\widehat{N} \subset \widehat{M}$ since $\Phi$ preserves injective morphisms. The image of $\pi_{[N]}$ is closed since it is proper. Then Proposition 4.5 implies that the fibre over $N \subset M$ is non-empty. So the image of $\pi_{[N]}$ contains $\mathcal{S}_{[N]}$, and then $\overline{\mathcal{S}_{[N]}}$. In particular, the fibre over a point of $\mathcal{S}_{[N]}$ reduces to a single point is the special case $U=N$ of Proposition 4.5.

Corollary 4.7. For arbitrary $M$ and e as above, the closure $\overline{\mathcal{S}_{[\widehat{N}]}}$ is an irreducible component of $\operatorname{Gr}_{\underline{\operatorname{dim}} \widehat{N}}(\widehat{M})$, and the map

$$
\pi=\bigsqcup_{[N] \in \operatorname{gsub}_{e}(M)} \pi_{[N]}: \bigsqcup_{[N] \in \operatorname{gsub}_{e}(M)} \overline{\mathcal{S}_{\widehat{N}}} \rightarrow \operatorname{Gr}_{e}(M)
$$

given by the restrictions of the $\pi_{[N]}$ to $\overline{\mathcal{S}_{\widehat{N}}}$ is a desingularization of $\operatorname{Gr}_{e}(M)$.

Proof. The stratum $\mathcal{S}_{[\widehat{N}]}$ of $\operatorname{Gr}_{\underline{\underline{d i m}} \widehat{N}}(\widehat{M})$ is irreducible and locally closed, of dimension

$$
\operatorname{dim} \operatorname{Hom}_{\Gamma}(\widehat{N}, \widehat{M})-\operatorname{dim} \operatorname{End}_{\Gamma}(\widehat{N})=\operatorname{dim} \operatorname{Hom}_{\Gamma}(\widehat{N}, \widehat{M} / \widehat{N})=\operatorname{dim} \operatorname{Gr}_{\underline{\underline{d i m}} \widehat{N}}(\widehat{M})
$$


as in the proof of [20, Proposition 7.1] since $\operatorname{Ext}^{1}(\widehat{N}, \widehat{N})=0$, so its closure is a connected (irreducible) component of $\mathrm{Gr}_{\underline{\operatorname{dim}} \widehat{N}}(\widehat{N})$ and thus a smooth variety. The image of this component under $\pi_{[N]}$ is thus an irreducible closed subvariety of $\operatorname{Gr}_{e}(M)$ containing its irreducible component $\overline{\mathcal{S}_{[N]}}$, and thus it equals $\overline{\mathcal{S}_{[N]}}$. Together with the other properties of the previous theorem, this implies that $\pi$ is a desingularization.

Remark 4.8. Following [20, Remark 7.8], we conjecture that $\mathrm{Gr}_{\operatorname{dim} \widehat{N}}(\widehat{M})$ is actually irreducible. This would imply that the constructions of desingularizations of the previous corollaries could be unified to the map

$$
\pi=\bigsqcup_{[N] \in \operatorname{gsub}_{e}(M)} \pi_{[N]}: \bigsqcup_{[N] \in \operatorname{gsub}_{e}(M)} \operatorname{Gr}_{\underline{\underline{\operatorname{dim}}} \widehat{N}}(\widehat{M}) \rightarrow \operatorname{Gr}_{e}(M)
$$

being a desingularization.

Corollary 4.9. Let $\Lambda$ be a self-injective gentle algebra. Then the desingularization map $\pi$ defined in Corollary 4.7 is defined for any finite generated $\Lambda$-module $M$ and dimension vector $e$.

Proof. Since $\Lambda$ is self-injective, we get that $\bmod \Lambda=\operatorname{Gproj} \Lambda$. The statement follows from Corollary 4.7 immediately.

In Section 5, we can see that even for self-injective gentle algebras, their quiver Grassmannians can be singular varieties.

\section{EXAMPLES}

5.1. Self-injective gentle Nakayama algebras. Let $Q$ be the following left quiver and $\Lambda=K Q /\langle\beta \alpha, \gamma \beta, \alpha \gamma\rangle$. Then its Cohen-Macaulay Auslander algebra $\Gamma=K Q^{\text {Aus }} /\left\langle I^{\text {Aus }}\right\rangle$, where $Q^{\text {Aus }}$ is as the following right quiver shows, and the ideal $I^{\text {Aus }}=\left\{\beta^{+} \alpha^{-}, \gamma^{+} \beta^{-}, \alpha^{+} \gamma^{-}\right\}$.
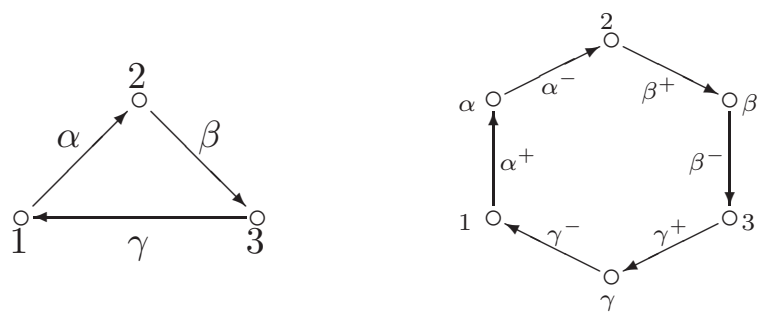

Figure 4. The quiver of $\Lambda$ and its Cohen-Macaulay Auslander algebra.

Let $M=P_{3} \oplus P_{3} \oplus S_{1} \oplus S_{3}$. Let $e=\underline{\operatorname{dim}} S_{3}+2 \underline{\operatorname{dim}} S_{1}$. We consider the quiver Grassmannian $X=\operatorname{Gr}_{e}(M)$. For simplicity, we restrict our attention to the full subquiver of $Q$ formed by vertices 1,3 . Choosing appropriate basis, the representation $M$ can be written as

$$
K^{3} \stackrel{\left(\begin{array}{lll}
1 & 0 & 0 \\
0 & 1 & 0 \\
0 & 0 & 0
\end{array}\right)}{\longleftarrow} K^{3} .
$$

In this way, the dimension vector $e$ equals $(2,1)$, thus, identifying $\operatorname{Gr}_{2}\left(K^{3}\right)$ with $\mathbb{P}^{2}$, the quiver Grassmannian $X$ can be realized as

$$
\left\{\left(\left(a_{0}: a_{1}: a_{2}\right),\left(b_{0}: b_{1}: b_{2}\right)\right) \in \mathbb{P}^{2} \times \mathbb{P}^{2} \mid a_{0} b_{0}+a_{1} b_{1}=0\right\},
$$

which is a singular projective variety of dimension three. 
We also restrict our attention to the full subquiver of $Q^{A u s}$ formed by vertices $1,3, \gamma$. The $\Gamma$-module $\widehat{M}=\Phi(M)$ admits the following explicit form:

$$
K^{3} \stackrel{\left(\begin{array}{ll}
1 & 0 \\
0 & 1 \\
0 & 0
\end{array}\right)}{\longleftarrow} K^{2} \stackrel{\left(\begin{array}{lll}
1 & 0 & 0 \\
0 & 1 & 0
\end{array}\right)}{\longleftarrow} K^{3} .
$$

The only generic subrepresentation type being $N=P_{3} \oplus S_{1}$, we thus have to consider subrepresentations of dimension vector $\underline{\operatorname{dim}} \widehat{N}=2 \underline{\operatorname{dim}} S_{1}+\underline{\operatorname{dim}} S_{3}+\underline{\operatorname{dim}} S_{\gamma}$ of $\widehat{M}$. Let $Y=\operatorname{Gr}_{\underline{\operatorname{dim}} \widehat{N}}(\widehat{M})$. Then the quiver Grassmannian $Y$ can be realized as

$$
\begin{gathered}
\left\{\left(\left(a_{0}: a_{1}: a_{2}\right),\left(c_{0}: c_{1}\right),\left(b_{0}: b_{1}: b_{2}\right) \in \mathbb{P}^{2} \times \mathbb{P}^{1} \times \mathbb{P}^{2} \mid\right.\right. \\
\left.a_{0} c_{0}+a_{1} c_{1}=0, b_{1} c_{0}=b_{0} c_{1}\right\},
\end{gathered}
$$

with the desingularization map being the projection to the first and third components.

5.2. Cluster-tilted algebra of type $\mathbb{A}_{n}$. Let $Q$ be the quiver as the following left diagram shows. Let $I=\left\{\alpha_{3} \alpha_{2}, \alpha_{2} \alpha_{1}, \alpha_{1} \alpha_{3}, \alpha_{5} \alpha_{4}, \alpha_{6} \alpha_{5}, \alpha_{4} \alpha_{6}\right\}$. Then $\Lambda=K Q /\langle I\rangle$ is a cluster-tilted algebra of type $\mathbb{A}_{5}$. Let $\Gamma=\operatorname{Aus}(\operatorname{Gproj} \Lambda)$ be the Cohen-Macaulay Auslander algebra. Then its quiver is as the following right diagram shows.
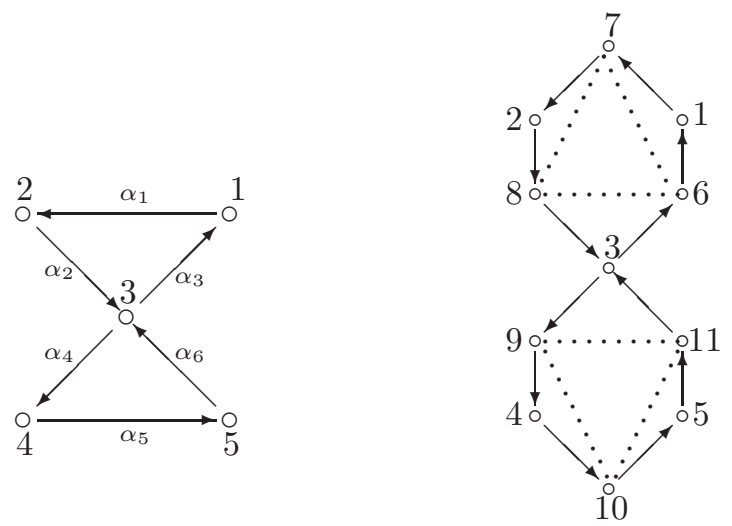

Figure 5. Cluster-tilted algebra of type $\mathbb{A}_{5}$ and its Cohen-Macaulay Auslander algebra.

Let $M=P_{3} \oplus R\left(\alpha_{2}\right) \oplus R\left(\alpha_{6}\right)$. Then $M$ is a Gorenstein projective module. Let $e=$ $\underline{\operatorname{dim}} P_{3}+\underline{\operatorname{dim}} S_{1}+\underline{\operatorname{dim}} S_{4}$. We consider the quiver Grassmannian $X=\operatorname{Gr}_{e}(M)$. For simplicity, we restrict our attention to the full subquiver of $Q$ formed by vertices $1,3,4$. Choosing appropriate basis, the representation $M$ can be written as

$$
K^{3} \stackrel{\left(\begin{array}{lll}
0 & 0 & 0 \\
0 & 1 & 0 \\
0 & 0 & 1
\end{array}\right)}{\longleftarrow} K^{3} \stackrel{\left(\begin{array}{lll}
1 & 0 & 0 \\
0 & 0 & 0 \\
0 & 0 & 1
\end{array}\right)}{\longrightarrow} K^{3} .
$$

In this way, the dimension vector $e$ equals $(2,1,2)$, thus, identifying $\operatorname{Gr}_{2}\left(K^{3}\right)$ with $\mathbb{P}^{2}$, the quiver Grassmannian $X$ can be realized as

$$
\left\{\left(\left(a_{0}: a_{1}: a_{2}\right),\left(b_{0}: b_{1}: b_{2}\right),\left(c_{0}: c_{1}: c_{2}\right)\right) \in \mathbb{P}^{2} \times \mathbb{P}^{2} \times \mathbb{P}^{2} \mid a_{1} b_{1}+a_{2} b_{2}=0, b_{0} c_{0}+b_{2} c_{2}=0\right\},
$$

which is a singular projective variety of dimension four.

We also restrict our attention to the full subquiver of $Q^{\text {Aus }}$ formed by vertices $1,3,4,6,9$. The $\Gamma$-module $\widehat{M}=\Phi(M)$ admits the following explicit form:

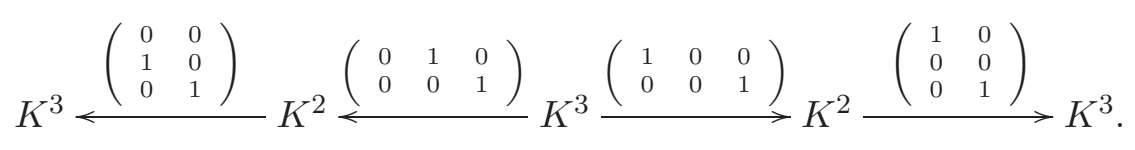


The only generic subrepresentation type being $N=P_{3} \oplus S_{1} \oplus S_{4}$, we thus have to consider subrepresentations of dimension vector $\underline{\operatorname{dim}} \widehat{N}=2 \underline{\operatorname{dim}} S_{1}+2 \underline{\operatorname{dim}} S_{4}+\underline{\operatorname{dim}} S_{3}+\underline{\operatorname{dim}} S_{6}+\underline{\operatorname{dim}} S_{9}$ of $\widehat{M}$. Let $Y=\operatorname{Gr}_{\underline{\underline{d i m}} \widehat{N}}(\widehat{M})$. Then the quiver Grassmannian $Y$ can be realized as

$$
\begin{gathered}
\left\{\left(\left(a_{0}: a_{1}: a_{2}\right),\left(d_{0}: d_{1}\right),\left(b_{0}: b_{1}: b_{2}\right),\left(e_{0}: e_{1}\right),\left(c_{0}: c_{1}: c_{2}\right)\right) \in \mathbb{P}^{2} \times \mathbb{P}^{1} \times \mathbb{P}^{2} \times \mathbb{P}^{1} \times \mathbb{P}^{2} \mid\right. \\
\left.d_{0} b_{2}=d_{1} b_{1}, b_{0} e_{1}=b_{2} e_{0}, a_{1} d_{0}+d_{2} d_{1}=0, c_{0} e_{0}+c_{2} e_{1}=0\right\},
\end{gathered}
$$

with the desingularization map being the projection to the first, third and fifth components.

\section{REFERENCES}

[1] I. Assem, T. Brüstle, G. Charbonneau-Jodoin and P. Plamondon, Gentle algebras arising from surface triangulations. Algebra Number Theory 4(2)(2010), 201-229.

[2] I. Assem and D. Happel, Generalized tilted algebras of type $\mathbb{A}_{n}$. Comm. Algebra 9(20)(1981), 21012125 .

[3] I. Assem and A. Skowroński, Iterated tilted algebras of type $\tilde{\mathbb{A}}_{n}$. Math. Z. 195(1987), 269-290.

[4] M. Auslander and M. Bridger, Stable module theory. Mem. Amer. Math. Soc. 94., Amer. Math. Soc., Providence, R.I., 1969.

[5] M. Auslander and I. Reiten, Application of contravariantly finite subcategories. Adv. Math. 86(1)(1991), 111-152.

[6] M. Auslander and I. Reiten, Cohen-Macaulay and Gorenstein artin algebras. In: Progress in Math. 95, Birkhäuser Verlag, Basel, 1991, 221-245.

[7] A. Beligiannis, Cohen-Macaulay modules, (co)tosion pairs and virtually Gorenstein algebras. J. Algebra 288(1)(2005), 137-211.

[8] A. Beligiannis, On algebras of finite Cohen-Macaulay type. Adv. Math. 226(2011), 1973-2019.

[9] C. Bessenrodt and T. Holm, $q$-Cartan matrices and combinatorial invariants of derived categories fro skewed-gentle algebras. Pac. J. Math. 229(1)(2007), 25-48.

[10] K. Bongartz, On degenerations and extensions of finite dimensional modules. Adv. Math. 121(1996), 245-287.

[11] A. B. Buan, R. Marsh and I. Reiten, Cluster-tilted algebras. Trans. Amer. Math. Soc. 359(2007), 323-332.

[12] R. Buchweitz, Maximal Cohen-Macaulay modules and Tate cohomology over Gorenstein Rings. Unpublished Manuscript, 1987. Available at Http://hdl.handle.net/1807/16682.

[13] I. Burban, Derived categories of coherent sheaves on rational singular curves, in: Representations of finite dimensional algebras and related topics in Lie Theory and geometry, Fields Inst. Commun. 40, Amer. Math. Soc., Providence, RI (2004), 173-188.

[14] M. C. R. Butler and C. M. Ringel, Auslander-Reiten sequences with few middle terms and applications to string algebras. Comm. Algebra 15(1987), 145-179.

[15] P. Caldero and F. Chapoton, Cluster algebras as Hall algebras of quiver representations. Comment. Maht. Helv. 81(3)(2006), 595-616.

[16] P. Caldero and B. Keller, From triangulated categories to cluster algebras. Inv. Math. 172(2008), 169-211.

[17] P. Caldero and M. Reineke, On the quiver Grassmannians in the acyclic case. J. Pure Appl. Algebra 212(11)(2008), 2369-2380.

[18] G. Cerulli Irelli, E. Feigin and M. Reineke, Quiver Grassmannians and degenerate flag varieties. Algebra and Number Theory 6(2012), 165-193.

[19] G. Cerulli Irelli, E. Feigin and M. Reineke, Degenerate flag varieties: moment graphs and Schröder numbers. J. Algebraic Combin. 38(2013), 159-189.

[20] G. Cerulli Irelli, E. Feigin and M. Reineke, Desingularization of quiver Grassmannians for Dynkin quivers. Adv. Math. 245(1)(2013), 182-207.

[21] X. Chen, S. Geng and M. Lu, The singularity categories of the cluster-tilted algebras of Dynkin type. Algebr. Represent. Theor. 18(2)(2015), 531-554.

[22] X. Chen and M. Lu, Singularity categories of skewed-gentle algebras. To appear in Colloquium Mathematicum.

[23] X. Chen and M. Lu, Cohen-Macaulay Auslander algebras of gentle algebras. Preprint, available at arXiv:1502.03948 math.RT]. 
[24] W. W. Crawley-Boevey, Maps between representations of zero-relation algebras. J. Algebra 126(2)(1989), 259-263.

[25] H. Derksen, J. Weyman and A. Zelevinsky, Quivers with potentials and their representations II: Applications to cluster algebras. J. Amer. Math. Soc. 23(2010), 749-790.

[26] E. E. Enochs and O. M. G. Jenda, Gorenstein injective and projective modules. Math. Z. 220(4)(1995), 611-633.

[27] E. Feigin, Degenerate flag varieties and the median Genochi nubmers, Math. Res. Lett. 18(6)(2011), 1163-1178.

[28] E. Feigin, $\mathbb{G}_{a}^{M}$ degeneration of flag varieties. Selecta Math. (N.S.) 18(3)(2012), 513-537.

[29] E. Feigin and M. Finkelberg, Degenerate flag varieties of type a: Frobenius splitting and BW theorem. Math. Z. 275(1-2)(2013), 55-77.

[30] S. Fomin and A. Zelevinsky, Cluster algebras I: Foundations, J. Amer. Math. Soc. 15(2)(2002), 497-529.

[31] C. Geiß and I. Reiten, Gentle algebras are Gorenstein. in Representations of algebras and related topics, Fields Inst. Commun. 45, Amer. Math. Soc., Providence, RI (2005), 129-133.

[32] D. Happel, On Gorenstein algebras. In: Representation Theory of finite groups and finite-dimensional Algebras, Progress in Math. 95, Birkhäuser Verlag, Basel, 1991, 389-404.

[33] D. Hernandez and Bernard Leclerc, Cluster algebras and quantum affine algebras. Duke Math. J. 154(2)(2010), 265-341.

[34] M. Kalck, Singularity categories of gentle algebras. Bull. London Math. Soc. 47(1)(2015), 65-74.

[35] B. Keller, On triangulated orbit categories. Doc. Math. 10(2005), 551-581.

[36] B. Keller and S. Scherotzke, Graded quiver varieties and derived categories. J. reine. angrew. Math., DOI 10.1515/crelle-2013-0124.

[37] B. Keller and S. Scherotzke, Desingularization of quiver Grassmannians via graded quiver varieties. Adv. Math. 256, 318-347.

[38] B. Leclerc and P. Plamondon, Nakajima varieties and repetitive algebras. Preprint, available at arXiv:1208:3910[math.QA]. To appear in Publ. RIMS, Kyoto.

[39] Z. W. Li, P. Zhang, Gorenstein algebras of finite Cohen-Macaulay type. Adv. Math. 223 (2010), 728734.

[40] H. Nakajima, Quiver varieties and finite-dimensional representations of quantum affine algebras. J. Amer. Math. Soc. 14(1)(2001), 145-238(electronic).

[41] H. Nakajima, Quiver varieties and cluster algebras. Kyoto Journal of Mathematics 51(1)(2011), 71-126.

[42] D. Orlov, Triangulated categories of singularities and D-branes in Landau-Ginzburg models. Proc. Steklov Inst. Math. 246(3)(2004), 227-248.

[43] S. Pan, Derived equivalences for Cohen-Macaulay Auslander algebras. J. Pure Appl. Algebra 216(2012), 355-363.

[44] M. Reineke, Every projective variety is a quiver Grassmannian. Algebr. Represent. Theory 16(5)(2013), 1313-1314.

[45] J. Rickard, Derived categories and stable equivalences. J. Pure Appl. Algebra 61(1989), 303-317.

[46] C. M. Ringel, The indecomposable representations of the dihedral 2-groups. Math. Ann. 214(1975), 19-34.

[47] A. Schofield, Generic representations of quivers. Proc. London Math. Soc. 65(3)(1992), 46-64.

[48] J. Schröer and A. Zimmermann, Stable endomoprhism algebras of modules over special biserial algebras. Preprint(2001), www.maths.leeds.ac.uk/ jschroer/preprints/dergen.ps.

[49] A. Skowroński and J. Waschbüsch, Representation-finite biserial algebras. J. Reine Angew. Math. 345(1983), 172-181.

[50] B. Wald and J. Waschbüsch, Tame biserial algebras. J. Algebra 95(1985), 480-500.

Department of Mathematics, Southwest Jiaotong University, Chengdu 610031, P.R.China

E-mail address: chenxinhong@swjtu.edu.cn

Department of Mathematics, Sichuan University, Chengdu 610064, P.R.China

E-mail address: luming@scu.edu.cn 